\title{
The history of mass dispersal around Herbig Ae/Be stars
}

\author{
A. Fuente ${ }^{1}$, J. Martín-Pintado ${ }^{1}$, R. Bachiller ${ }^{1}$, A. Rodríguez-Franco ${ }^{2}$, and F. Palla ${ }^{3}$ \\ 1 Observatorio Astronómico Nacional (IGN), Campus Universitario, Apdo. 1143, 28800 Alcalá de Henares, \\ Madrid, Spain \\ 2 Escuela Universitaria de Optica, Departamento de Matemática Aplicada (Biomatemática), Av. Arcos \\ de Jalón s/n, 28037 Madrid, Spain \\ 3 Osservatorio Astrofisico di Arcetri, Largo Enrico Fermi, 550125 Firenze, Italy
}

\author{
Received 4 December 2001 / Accepted 21 March 2002
}

\begin{abstract}
We present a systematic study of the material surrounding intermediate-mass stars. Our sample includes 34 Herbig Ae/Be (HAEBE) stars of different ages and luminosities. This is a quite complete representation of the whole class of HAEBE stars and consequently, our conclusions should have a solid statistical meaning. In addition, we have observed 2 intermediate-mass protostars and included published data on 15 protostellar objects in order to determine the evolution of the circumstellar material in the early stages of stellar evolution. All the HAEBE stars have been classified according with the three Types already defined in Fuente et al. (1998): Type I stars are immersed in a dense clump and have associated bipolar outflows, their ages are $\sim 0.1$ Myr; Type II stars are still immersed in the molecular cloud though not in a dense clump, their ages are between $\sim$ a few 0.1 to $\sim \mathrm{a}$ few Myr; Type III stars have completely dispersed the surrounding material and are located in a cavity of the molecular cloud, their ages are $>1$ Myr. Our observations are used to reconstruct the evolution of the circumstellar material around intermediate-mass stars and investigate the mass dispersal mechanisms at the different stages of the stellar evolution. Our results can be summarized as follows: intermediate-mass stars disperse $\geq 90 \%$ of the mass of the parent clump during the protostellar phase. During this phase, the energetic outflows sweep out the gas and dust forming a biconical cavity while the equatorial material is infalling to feed the circumstellar disk and eventually the protostar. In this way, the density structure of the parent clump remains well described by a density law $n \propto r^{\beta}$ with $-2<\beta<-1$ although a large fraction of the mass is dispersed. In $\sim$ a few 0.1 Myr, the star becomes visible and the outflow fades. Some material is dispersed from $\sim$ a few 0.1 to $\geq 1 \mathrm{Myr}$. Since the outflow declines and the stars are still too cold to generate UV photons, stellar winds are expected to be the only dispersal mechanism at work. In $1 \mathrm{Myr}$ an early-type star (B0-B5) and in $\geq 1$ to $10 \mathrm{Myr}$ a late-type star (later than B6) meets the ZAMS. Now the star is hot enough to produce UV photons and starts excavating the molecular cloud. Significant differences exist between early-type and late-type stars at this evolutionary stage. Only early-type stars are able to create large $(R>0.08 \mathrm{pc})$ cavities in the molecular cloud, producing a dramatic change in the morphology of the region. This difference is easily understood if photodissociation plays an important role in the mass dispersal around these objects.
\end{abstract}

Key words. stars: formation - stars: pre-main sequence - ISM: abundances - ISM: molecules

\section{Introduction}

The evolutionary stage of a young star is closely related to the distribution of its circumstellar material. Star formation begins when a dense and cold fragment collapses forming a hydrostatic core surrounded by a massive envelope that hides the central object. Early in the evolution, powerful stellar winds are developed and the envelope begins to be dispersed. Fuente et al. (1998) (hereafter Paper I) have proposed that in a typical time of $10 \mathrm{Myr}$ a low mass star and $1 \mathrm{Myr}$ an intemediate-mass

Send offprint requests to: A. Fuente,

e-mail: fuente@oan.es star disperses the surrounding envelope. The study of the details of this dispersal (energetics, timescales...) and the investigation of the possible dispersal mechanisms in intermediate-mass stars constitute the aim of this paper.

HAEBE stars are intermediate-mass stars old enough to be optically visible but still associated with the parent molecular cloud. An initial attempt to study the process of circumstellar mass dispersal in these objects was carried out by Hillenbrand et al. (1992) who studied the spectral energy distribution of 47 HAEBE stars. Hillenbrand proposed a classification scheme based on the infrared spectral index in three groups ordered by a progressive decrease of circumstellar material from 
Group II $\rightarrow$ Group I $\rightarrow$ Group III. However, owing to the heterogeneous group of HAEBE stars, she could not find a correlation between the stellar ages and the IR groups.

Infrared continuum observations only trace the warm dust very close to the star and suffer from important opacity effects. Hillenbrand (1995) mapped in molecular lines the clouds associated with 17 Herbig Ae/Be (HAEBE) stars and found morphologies which revealed that the cloud is being disrupted by the young star. The moderate resolution of these observations $(H P B W \sim 45 \operatorname{arcsec}$ at $2.6 \mathrm{~mm}$ ) did not allow a more detailed study of the mass dispersal around the star. We carried out a high angular resolution study of the environment of $14 \mathrm{Herbig} \mathrm{Ae} / \mathrm{Be}$ (HAEBE) stars in molecular lines and continuum emission at millimeter wavelenghts (Paper I). Continuum emission at millimeter wavelengths is expected to be optically thin even towards the more deeply embedded objects and to trace dust as cold as $T_{\mathrm{d}} \sim 10 \mathrm{~K}$. Our data showed that the circumstellar gas and dust is progressively dispersed by the HAEBE star during their evolution to the mainsequence and that the efficiency of this dispersal depends on the spectral type of the star. While around early-type stars (B0-B5) the mean gas density in a radius of $0.08 \mathrm{pc}$ decreases by almost two orders of magnitude prior to the main-sequence, in late-type stars (later than B6) it decreases by less than an order of magnitude. Moreover, HAEBE stars disperse the circumstellar dense gas in such a way that the morphology of the parent molecular cloud is correlated with the age of the stars. Based on this correlation, we proposed a new classification for HAEBE stars: Type I stars are immersed in a dense clump, have associated bipolar outflows, and their ages are $\sim 10^{5}$ years. In Type III stars, the surrounding dense gas has been completely dispersed and the central star is located in a large cavity. There are no bipolar outflows and their ages are $>10^{6}$ years. Finally, Type II stars represent the intermediate case, still immersed in the molecular cloud but not at the peak of a dense clump. The advantage of this new classification is that it allows a simple and independent estimate of the age of the star.

In this paper, we study the dense gas around a larger sample of 34 HAEBE stars and classify them according to its spatial distribution. Our sample is representative of the class of HAEBE stars so that our conclusions have a solid statistical basis. In addition, we have observed 2 and compiled data on 15 intermediate-mass protostars in order to study the onset of mass dispersal before the star becomes visible. Taking into account these new results and recent papers on this subject (Fuente et al. 2001; Bachiller et al. 2002; Font et al. 2001), we reconstruct the history of mass dispersal in intermediate-mass stars and extend and update the discussion of Paper I.

\section{Selected sample}

Our initial sample has been enlarged to include 34 HAEBE stars and the two protostars NGC 7129 - FIRS 2 and RNO6 - NW (see Table 1). HAEBE stars have been selected to include 14 early-type and 20 late-type stars of different ages, from $<0.1$ to $8 \mathrm{Myr}$. Most of the selected stars are at distances between 500 and $1500 \mathrm{pc}$ in order to have good spatial resolution. The coordinates listed in Table 1 are the $(0,0)$ position of our maps. They have been taken from Thé et al. (1994) and Herbig \& Bell (1988). Recent star catalogues (Perryman et al. 1997; Hog et al. 1998) give new positions for some stars which differ by $\sim 1$ arcsecond from those listed in Table 1 . This difference is not relevant for our study since it constitutes $<10 \%$ of the Half Power Beam Width $(H P B W)$ of our observations. For the stars in our initial sample, the stellar ages have been taken from Paper I. For the new stars, we have calcualted the ages using the evolutionary tracks and isochrones from Palla \& Stahler (1993) and the luminosities and effective temperatures given in Table 1.

The far-infrared source NGC 7129-FIRS2 is located in the bright rim which surrounds the reflection nebula NGC 7129, and is associated with a powerful outflow (Bechis et al. 1978; Eiroa et al. 1998; Fuente et al. 2001). NGC 7129-FIRS 2 has not been detected in the optical and the near-infrared. Eiroa et al. (1998) derived a luminosity of $430 L_{\odot}$, a dust temperature of $35 \mathrm{~K}$ and a mass of $6 M_{\odot}$. Measurements by Font et al. (2001) are consistent with these results. The low dust temperature and $L_{\mathrm{bol}} / L_{1.3 \mathrm{~mm}}$ ratio of this source suggest that it is an intermediate mass counterpart of Class 0 low-mass stars. Interferometric millimeter continuum observations reported by Fuente et al. (2001) suggest that the far infrared source could be in fact a cluster of protostars. Testi et al. (1998) concluded that clustering is significant only for stars of spectral type earlier than B7. Given the high luminosity of NGC 7129-FIRS 2 and the clustering condition found by Fuente et al. (2001), we consider that this source could be a precursor of an early-type Be star.

Bachiller et al. (2002) detected an energetic bipolar outflow associated with the far infrared source IRAS $02124+5514$ (RNO $6 \mathrm{NW}$ ). This source presents an increasing spectrum from 12 to $100 \mu \mathrm{m}$ and is not detected at optical wavelenghts. With a luminosity of $>27 L_{\odot}$, this protostar could be a precursor of a Herbig Ae star.

In Table 2 we list values of the circumstellar mass $(R \leq$ $0.08 \mathrm{pc}$ ) and outflow parameters found in the literature for the sample of 15 protostars. These protostars have been selected for being well-studied objects, cover a wide range of luminosities (from $\sim 10$ to a few $10^{4} L_{\odot}$ ) and be located at a distance $<2 \mathrm{kpc}$.

\section{Observations}

The observations were carried out in June 1998 using the IRAM 30m telescope in Pico de Veleta (Spain). All the HAEBE stars were observed in the ${ }^{13} \mathrm{CO} 1 \rightarrow 0, \mathrm{C}^{18} \mathrm{O} 1 \rightarrow 0$ and ${ }^{12} \mathrm{CO} 2 \rightarrow 1$ lines. The Half Power Beam Width of the telescope is $24^{\prime \prime}$ at the frequency of ${ }^{13} \mathrm{CO} 1 \rightarrow 0$ and $\mathrm{C}^{18} \mathrm{O}$ $1 \rightarrow 0$ lines and $12^{\prime \prime}$ at the frequency of the ${ }^{12} \mathrm{CO} 2 \rightarrow 1$ line. The three lines could be observed simultaneously taking advantage of the multi-receiver capability of the telescope. 
Table 1. List of objects.

\begin{tabular}{|c|c|c|c|c|c|c|c|c|}
\hline \multicolumn{9}{|c|}{ Spectral types B0-B5 } \\
\hline Object & RA (1950) & Dec (1950) & Sp. type & $d(\mathrm{pc})$ & $\log L\left(L_{\odot}\right)$ & $\log T(\mathrm{~K})$ & Age (Myr) & Ref \\
\hline NGC 7129 - FIRS 2 & $21^{\mathrm{h}} 41^{\mathrm{m}} 52^{\mathrm{s}} .1$ & $+65^{\circ} 49^{\prime} 35^{\prime \prime} .5$ & B5 & 1250 & 2.63 & & $<0.1$ & 6 \\
\hline $\mathrm{LkH} \alpha 234$ & $21^{\mathrm{h}} 41^{\mathrm{m}} 57^{\mathrm{s}} \cdot 6$ & $+65^{\circ} 53^{\prime} 07^{\prime \prime} 1$ & B5-7 & 1250 & $2.45,2.74$ & $4.09,4.14$ & $\sim 0.1$ & 1 \\
\hline MWC 1080 & $23^{\mathrm{h}} 15^{\mathrm{m}} 14^{\mathrm{s}} .8$ & $+60^{\circ} 34^{\prime} 19^{\prime \prime} .2$ & B0 & 1000 & 4.59 & 4.49 & $<1$ & 2 \\
\hline RNO 6 & $02^{\mathrm{h}} 13^{\mathrm{m}} 03^{\mathrm{s}} .0$ & $+55^{\circ} 09^{\prime} 03^{\prime \prime} .0$ & B1 & 1600 & 2.34 & 4.35 & & \\
\hline HD 259431 & $06^{\mathrm{h}} 30^{\mathrm{m}} 19^{\mathrm{s}} .4$ & $+10^{\circ} 21^{\prime} 38^{\prime \prime} .2$ & B5 & 800 & 3.16 & 4.14 & 0.1 & \\
\hline MWC 137 & $06^{\mathrm{h}} 15^{\mathrm{m}} 53.6$ & $+15^{\circ} 18^{\prime} 06^{\prime \prime} .9$ & B0 & 1300 & 4.46 & 4.49 & $<1$ & 2 \\
\hline HD 53367 & $07^{\mathrm{h}} 02^{\mathrm{m}} 03^{\mathrm{s}} .6$ & $-10^{\circ} 22^{\prime} 43^{\prime \prime} .7$ & B0 & 1150 & $3.37-4.26$ & 4.50 & & 1 \\
\hline VV Ser & $18^{\mathrm{h}} 26^{\mathrm{m}} 14^{\mathrm{s}} .3$ & $+00^{\circ} 06^{\prime} 39^{\prime \prime} 6$ & B0-9 & 440 & 2.03 & 4.03 & 0.6 & \\
\hline MWC 297 & $18^{\mathrm{h}} 25^{\mathrm{m}} 01^{\mathrm{s}} .4$ & $-03^{\circ} 51^{\prime} 47^{\prime \prime} 0$ & O9 & 450 & 4.60 & 4.52 & & \\
\hline HD 200775 & $21^{\mathrm{h}} 00^{\mathrm{m}} 59.7$ & $+67^{\circ} 57^{\prime} 55^{\prime \prime} .5$ & B2.5 & 430 & 3.89 & 4.31 & 8 & 3 \\
\hline $\mathrm{BD}+651637$ & $21^{\mathrm{h}} 41^{\mathrm{m}} 41^{\mathrm{s}} .1$ & $+65^{\circ} 52^{\prime} 49^{\prime \prime} 0$ & B3 & 1250 & 3.38 & 4.25 & 5 & 2,4 \\
\hline HD 216629 & $22^{\mathrm{h}} 51^{\mathrm{m}} 18^{\mathrm{s}} .4$ & $+61^{\circ} 52^{\prime} 46^{\prime \prime} 0$ & $\mathrm{~B} 2$ & 725 & 3.85 & 4.31 & & \\
\hline BHJ 71 & $23^{\mathrm{h}} 03^{\mathrm{m}} 07^{\mathrm{s}} .0$ & $+61^{\circ} 59^{\prime} 36^{\prime \prime} .0$ & B0 & 730 & 4.06 & 4.49 & & \\
\hline HD 37490 & $05^{\mathrm{h}} 36^{\mathrm{m}} 32^{\mathrm{s}} .6$ & $+04^{\circ} 05^{\prime} 40^{\prime \prime} 6$ & $\mathrm{~B} 2$ & 360 & 3.95 & 4.25 & & \\
\hline HD 52721 & $06^{\mathrm{h}} 59^{\mathrm{m}} 28^{\mathrm{s}} .6$ & $-11^{\circ} 13^{\prime} 41^{\prime \prime} .4$ & $\mathrm{~B} 2$ & 450 & 3.82 & 4.35 & 5 & 4 \\
\hline \multicolumn{9}{|c|}{ Spectral types later than B6 } \\
\hline Object & RA (1950) & Dec (1950) & Sp. type & $d(\mathrm{pc})$ & $\log L\left(L_{\odot}\right)$ & $\log T(\mathrm{~K})$ & Age (Myr) & Ref \\
\hline RNO 6-NW & $02^{\mathrm{h}} 12^{\mathrm{m}} 32^{\mathrm{s}} .1$ & $+55^{\circ} 14^{\prime} 27^{\prime \prime} .0$ & A5 & 1600 & 1.43 & & $<0.1$ & \\
\hline PV Cep & $20^{\mathrm{h}} 45^{\mathrm{m}} 23.5$ & $+67^{\circ} 46^{\prime} 33^{\prime \prime} 0$ & A5 & 500 & 2.07 & 3.85 & 0.1 & 1 \\
\hline $\mathrm{Z} \mathrm{CMa}$ & $07^{\mathrm{h}} 01^{\mathrm{m}} 22^{\mathrm{s}} .5$ & $-11^{\circ} 28^{\prime} 36^{\prime \prime} 0$ & Bpec & 1150 & 3.85 & $3.80,4.00$ & & \\
\hline $\mathrm{LkH} \alpha 198$ & $00^{\mathrm{h}} 08^{\mathrm{m}} 47^{\mathrm{s}} .4$ & $+58^{\circ} 33^{\prime} 05^{\prime \prime} .2$ & A5 & 600 & $2.35,2.75$ & 3.99 & 0.1 & 1 \\
\hline Elias 1 & $04^{\mathrm{h}} 15^{\mathrm{m}} 34^{\mathrm{s}} .5$ & $+28^{\circ} 12^{\prime} 01^{\prime \prime} .8$ & A6 & 160 & $1.31-1.71$ & 3.91 & $2-5$ & 1 \\
\hline $\mathrm{LkH} \alpha 215$ & $06^{\mathrm{h}} 29^{\mathrm{m}} 56^{\mathrm{s}} 2$ & $+10^{\circ} 11^{\prime} 51^{\prime \prime} 0$ & B7 & 800 & $2.45,2.84$ & $4.05,4.09$ & 0.1 & 1 \\
\hline $\mathrm{LkH} \alpha 233$ & $22^{\mathrm{h}} 32^{\mathrm{m}} 28^{\mathrm{s}} \cdot 3$ & $+40^{\circ} 24^{\prime} 32^{\prime \prime} 2$ & A5 & 880 & $2.00,2.19$ & 3.90 & 0.5 & 1 \\
\hline $\mathrm{BD}+463471$ & $21^{\mathrm{h}} 50^{\mathrm{m}} 38^{\mathrm{s}} .5$ & $+46^{\circ} 59^{\prime} 35^{\prime \prime} .4$ & A0 & 900 & $2.30-2.71$ & 3.99 & $0.1-0.3$ & 1 \\
\hline HD 250550 & $05^{\mathrm{h}} 59^{\mathrm{m}} 06^{\mathrm{s}} .4$ & $+16^{\circ} 30^{\prime} 59^{\prime \prime} 4$ & B7 & 700 & 2.32 & 4.09 & 0.3 & \\
\hline AB Aur & $04^{\mathrm{h}} 52^{\mathrm{m}} 34^{\mathrm{s}} .2$ & $+30^{\circ} 28^{\prime} 21^{\prime \prime} .9$ & A0 & 160 & 1.86 & 3.99 & 5 & \\
\hline LKH $\alpha 208$ & $06^{\mathrm{h}} 04^{\mathrm{m}} 53.2$ & $+18^{\circ} 39^{\prime} 55^{\prime \prime} .0$ & $\mathrm{~A} 2$ & 1000 & 1.89 & 3.94 & 1.1 & \\
\hline RR Tau & $05^{\mathrm{h}} 36^{\mathrm{m}} 23.8$ & $+26^{\circ} 20^{\prime} 49^{\prime \prime} 2$ & A 3.5 & 800 & 1.78 & 3.93 & 0.7 & 2 \\
\hline Par 21 & $19^{\mathrm{h}} 26^{\mathrm{m}} 37^{\mathrm{s}} .5$ & $+09^{\circ} 32^{\prime} 32^{\prime \prime} 0$ & F8 & 1300 & 1.44 & 3.78 & 0.7 & \\
\hline $\mathrm{BD}+61154$ & $00^{\mathrm{h}} 40^{\mathrm{m}} 21^{\mathrm{s}} .9$ & $+61^{\circ} 38^{\prime} 15^{\prime \prime} .1$ & B8 & 650 & 2.52 & 4.05 & 0.7 & 1,2 \\
\hline BF Ori & $05^{\mathrm{h}} 34^{\mathrm{m}} 47^{\mathrm{s}} .2$ & $-06^{\circ} 36^{\prime} 45^{\prime \prime} .3$ & A7 & 460 & 1.53 & 3.90 & 2.8 & 7 \\
\hline HK Ori & $05^{\mathrm{h}} 28^{\mathrm{m}} 40^{\mathrm{s}} .1$ & $+12^{\circ} 07^{\prime} 00^{\prime \prime} .2$ & A 4 & 450 & $1.43,1.61$ & 3.93 & 5 & 1 \\
\hline T Ori & $05^{\mathrm{h}} 33^{\mathrm{m}} 23^{\mathrm{s}} .1$ & $-05^{\circ} 30^{\prime} 25^{\prime \prime} .9$ & $\mathrm{~A} 2$ & 450 & $1.89,1.92$ & 3.94 & 1.1 & 2 \\
\hline $\mathrm{LkH} \alpha 25$ & $06^{\mathrm{h}} 37^{\mathrm{m}} 59.5$ & $+09^{\circ} 50^{\prime} 53^{\prime \prime} 4$ & B7 & 800 & $1.83,2.93$ & 4.05 & 2 & \\
\hline $\mathrm{LkH} \alpha 218$ & $07^{\mathrm{h}} 00^{\mathrm{m}} 21^{\mathrm{s}} .9$ & $-11^{\circ} 21^{\prime} 46^{\prime \prime} 0$ & B9 & 1150 & 2.09 & 4.03 & 0.7 & \\
\hline $\mathrm{LkH} \alpha 257$ & $21^{\mathrm{h}} 52^{\mathrm{m}} 22^{\mathrm{s}} .8$ & $+46^{\circ} 57^{\prime} 58^{\prime \prime} .2$ & B8 & 900 & 1.85 & 4.05 & 2 & \\
\hline HD 245185 & $05^{\mathrm{h}} 32^{\mathrm{m}} 24^{\mathrm{s}} .1$ & $+09^{\circ} 59^{\prime} 57^{\prime \prime} .2$ & A1 & 400 & 1.34 & 3.96 & 6.4 & \\
\hline
\end{tabular}

Ref: (1) Berrilli et al. (1992); (2) Hillenbrand et al. (1992); (3) Hipparcos data; (4) van den Ancker, private communication; (5) Cabrit \& Bertout (1992); (6) Eiroa et al. (1998); (7) Testi et al. (1998).

Forward efficiency, main beam efficiency, and typical system temperature were $0.92,0.68$ and $350 \mathrm{~K}$ at $3 \mathrm{~mm}$; and $0.86,0.39$ and $400-700 \mathrm{~K}$ at $1.3 \mathrm{~mm}$. The backends were an autocorrelator split in several parts and a $256 \times 100 \mathrm{kHz}$ filter-bank. All the observations were carried out with a spectral resolution of $\sim 78 \mathrm{kHz}$. The intensity scale is the main brightness temperature. Maps of $\approx 200^{\prime \prime} \times 200^{\prime \prime}$ $\left(1 \mathrm{pc} \times 1 \mathrm{pc}\right.$ at a distance of $1000 \mathrm{pc}$ ) with a spacing of $20^{\prime \prime}$ were observed towards each star where molecular emission was detected. In some cases, the central region was fully sampled with a spacing of $10^{\prime \prime}$. In our final set of data, we have also included the observations presented in Paper I.

\section{Results}

The maps of the integrated line intensity of the ${ }^{13} \mathrm{CO}$ $J=1 \rightarrow 0$ line are shown in Figs. 1 and 2. In Fig. 1 we show a region of $1 \mathrm{pc} \times 1 \mathrm{pc}$ around the observed 12 earlytype stars. The protostar NGC 7129-FIRS 2 has not been included in this figure. This young object is invisible in 
Table 2. Protostars and outflows parameters.

\begin{tabular}{llllllll}
\hline \hline Object & $d(\mathrm{pc})$ & $L\left(L_{\odot}\right)$ & $M_{\mathrm{c}}\left(M_{\odot}\right)$ & $M_{\odot}\left(M_{\odot} \mathrm{yr}^{-1}\right)$ & Age $\left(10^{4}\right.$ yrs $)$ & $L_{\text {mech }}$ & Ref. \\
\hline NGC 2071 & 500 & 520 & 60 & $6 \times 10^{-4}$ & 1.6 & 4 & 1,2 \\
GL 490 & 900 & 1400 & 31 & $9 \times 10^{-4}$ & 1.7 & 6.8 & 1,2 \\
S 140 & 910 & 5000 & 85 & $2 \times 10^{-3}$ & 1.5 & 7.8 & 1,2 \\
IRAS 23385+6053 & 4900 & $1.6 \times 10^{4}$ & 350 & $>10^{-3}$ & 0.7 & 21.6 & 3 \\
IRAS 20126+4104 & 1700 & $\sim 10^{4}$ & $10-50$ & $810^{-4}$ & 6.4 & 5.9 & 4,15 \\
CB3 & 2500 & 930 & 80 & $3 \times 10^{-4}$ & 1.0 & 5.6 & 5,10 \\
HH7-11 & 350 & 75 & $4-13$ & $1 \times 10^{-4}$ & 1.0 & 2.6 & 6,7 \\
L1551-IRS 5 & 160 & 33 & $0.7-20$ & $6.7 \times 10^{-5}$ & 5.0 & 2.1 & 7,8 \\
IRAS 16293 & 160 & 23 & 2.5 & $5.6 \times 10^{-5}$ & 0.7 & 0.4 & 7,9 \\
L1455M-FIR & 350 & 16 & $2-28$ & $8 \times 10^{-5}$ & 2.9 & 0.3 & 7,8 \\
GL 2591 & 2000 & $2 \times 10^{4}$ & 613 & $6 \times 10^{-4}$ & 7.0 & 3.5 & 11 \\
Cep A & 725 & $5 \times 10^{4}$ & 391 & $1 \times 10^{-3}$ & 1.1 & 1.7 & 12 \\
Mon R2 & 950 & $5 \times 10^{4}$ & 178 & $3 \times 10^{-3}$ & 4.7 & 1.9 & 12 \\
G192.16 & 2000 & 1480 & $17-36$ & $5 \times 10^{-4}$ & 17 & 2.1 & 13 \\
GGD27-IRS & 1700 & $2 \times 10^{4}$ & $120-400$ & $5 \times 10^{-3}$ & 10 & 6 & 14 \\
\hline
\end{tabular}

References: 1) Snell et al. (1984); 2) From $\mathrm{H}^{13} \mathrm{CO}^{+}$unpublished data by Fuente et al. 3) Molinari et al. (1998); 4) Shepherd et al. (2000); 5) Codella \& Bachiller (1999); 6) Sandell et al. (1990); 7) Cabrit \& Bertout (1992); 8) Davidson (1987); 9) Mundy et al. (1986); 10) Laundhart et al. (1997); 11) Hasegawa \& Mitchell (1995); 12) Margulis \& Lada (1985); 13) Shepherd et al. (1998); 14) Yamashita et al. (1989); 15) Cesaroni et al. (1999).

the ${ }^{13} \mathrm{CO} J=1 \rightarrow 0$ and $\mathrm{C}^{18} \mathrm{O} J=1 \rightarrow 0$ lines probably because of depletion and opacity effects. However, an intense point source is detected in the $1.3 \mathrm{~mm}$ continuum emission. The integrated intensity maps of the ${ }^{13} \mathrm{CO} J=1 \rightarrow 0$ and $\mathrm{C}^{18} \mathrm{O} J=1 \rightarrow 0$ lines towards NGC 7129-FIRS 2, and the continuum image at $1.3 \mathrm{~mm}$ are shown in Fig. 3.

In Fig. 2 we show the ${ }^{13} \mathrm{CO} J=1 \rightarrow 0$ maps around the sample of 20 late-type stars. Because of the proximity of two stars $(d \sim 160 \mathrm{pc})$, we could not cover the same region of $1 \mathrm{pc} \times 1 \mathrm{pc}$. Thus, the maps presented in Fig. 2 have different linear sizes. In order to have a reference for the spatial scale, we have drawn a circle of $0.08 \mathrm{pc}$ of radius in each panel. Toward several stars we searched for molecular emission in a cross of $160^{\prime \prime} \times 160^{\prime \prime}$ $(\mathrm{LkH} \alpha 218), 240^{\prime \prime} \times 240^{\prime \prime}$ (HD 245185), 480"×480" (HD 37490 ) and $240^{\prime \prime} \times 240^{\prime \prime}$ (HD 52721). Since no molecular emission was detected, we did not include these stars in Figs. 1 and 2, but the results are included in Table 3.

In Figs. 1 and 2 we have ordered the stars following a tentative evolutionary sequence. In fact, a quick look shows a trend in which the youngest stars are immersed in dense cores, while the oldest ones are found in cavities created during the pre-main sequence evolution. In Paper I, we used two quantitative indicators of gas dispersal: the mass within a radius of $0.08 \mathrm{pc}$ around the star, $M_{R=0.08 \mathrm{pc}}$, and the spatial index $\alpha$ which defines the distribution of the total column density around the star. The radius of $0.08 \mathrm{pc}$ was not chosen arbitrarily, but corresponds to a beam size of $24^{\prime \prime}$ (the beam of the ${ }^{13} \mathrm{CO}$ $J=1 \rightarrow 0$ observations) at the distance of $1300 \mathrm{pc}$, that of MWC 137 the most distant object of the sample presented in Paper I. Although we have now included a more distant star, RNO $6(d=1600 \mathrm{pc})$, we have kept the reference radius of $0.08 \mathrm{pc}$ for consistency with our previous analysis.

\subsection{Mass around the star}

Unfortunately, it is not possible to use a unique tracer of the circumstellar mass for all the stars. The ${ }^{13} \mathrm{CO}$ $J=1 \rightarrow 0$ line traces low density $\left(\sim 10^{3} \mathrm{~cm}^{-3}\right)$ and cool gas $\left(T_{\mathrm{k}}<30 \mathrm{~K}\right)$, and is probably the best diagnostic in evolved objects which are located either in cavities or at the edges of molecular clouds. However, this line can severely underestimate the circumstellar mass in the less evolved objects where it is probably optically thick. Simultaneously with the ${ }^{13} \mathrm{CO} J=1 \rightarrow 0$ line, we have observed the $\mathrm{C}^{18} \mathrm{O} 1 \rightarrow 0$ line. The ratio between these two lines can give an estimate of the opacity of the ${ }^{13} \mathrm{CO} J=1 \rightarrow 0$ line. But for the youngest objects, even the $\mathrm{C}^{18} \mathrm{O} 1 \rightarrow 0$ line could be optically thick. On the other hand, recent studies have shown that both isotopes, ${ }^{13} \mathrm{CO}$ and $\mathrm{C}^{18} \mathrm{O}$, can be significantly depleted in the central parts of deeply embedded objects (Tafalla et al. 2002). In these cases, the observation of these molecular lines cannot be used to estimate the circumstellar mass and the dust continuum emission at $1.3 \mathrm{~mm}$ is preferable. For example, this is the case for NGC 7129-FIRS 2 (see Fig. 3).

All the mass tracers, the dust continuum emission and molecular lines, are measuring the total amount of mass in the beam of the telescope. We assume that the mass measured within a radius of $0.08 \mathrm{pc}$ around the star is truly circumstellar. However, because of projection effects we could have a contribution of more distant positions of the molecular cloud. The uncertainty due to this contribution is negligible towards the youngest objects in which 


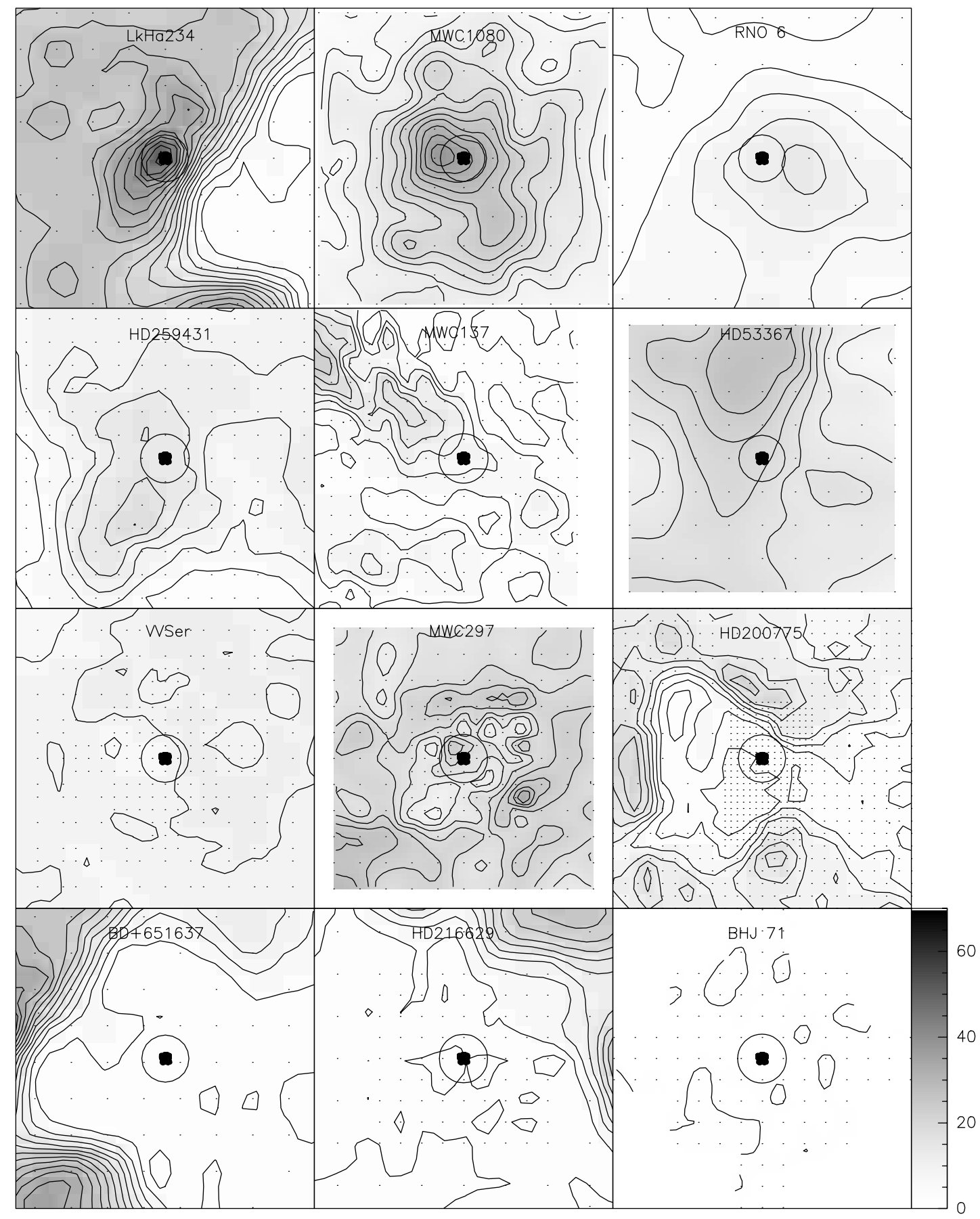

Fig. 1. Integrated intensity maps of the ${ }^{13} \mathrm{CO} J=1 \rightarrow 0$ line around the early-type HAEBE stars of our sample. The size of the box is $1 \mathrm{pc} \times 1 \mathrm{pc}$ for all the objects. Contour levels are 0.3 to 70 by $4 \mathrm{~K} \mathrm{~km} \mathrm{~s}^{-1}$. Points indicate the observed positions. The stars are ordered in a tentative evolutionary sequence in which the morphology of the cloud changes from a centrally peaked condensation (top) to a cavity (bottom), as the star evolves toward the main sequence.

as we will dicuss below the cloud density decays rapidly with the distance to the star. But it could be important in the case of more evolved stars. In these cases, the spatial index $\alpha$ will be a better estimate of the evolutionary stage of the star.

In Table 3, we list the values of the mass estimated from the molecular lines assuming optically thin emission, a rotation temperature of $30 \mathrm{~K}$, and standard values of the molecular abundances $\left[{ }^{13} \mathrm{CO} / \mathrm{H}_{2}\right]=2 \times 10^{-6}$ and
$\left[\mathrm{C}^{18} \mathrm{O} / \mathrm{H}_{2}\right]=2 \times 10^{-7}$ respectively (Dickman 1978 ). In this approximation, the computed masses are proportional to the integrated line intensities. The agreement within a factor of 2 between the masses derived from ${ }^{13} \mathrm{CO}$ and $\mathrm{C}^{18} \mathrm{O}$ shows that optical depth effects are not important in most sources. The assumed rotation temperature is uncertain by a factor of 2 (see Paper I and references therein) which translates in an uncertainty of a factor of 2 in the mass estimate. Masses from the $1.3 \mathrm{~mm}$ dust continuum emission 


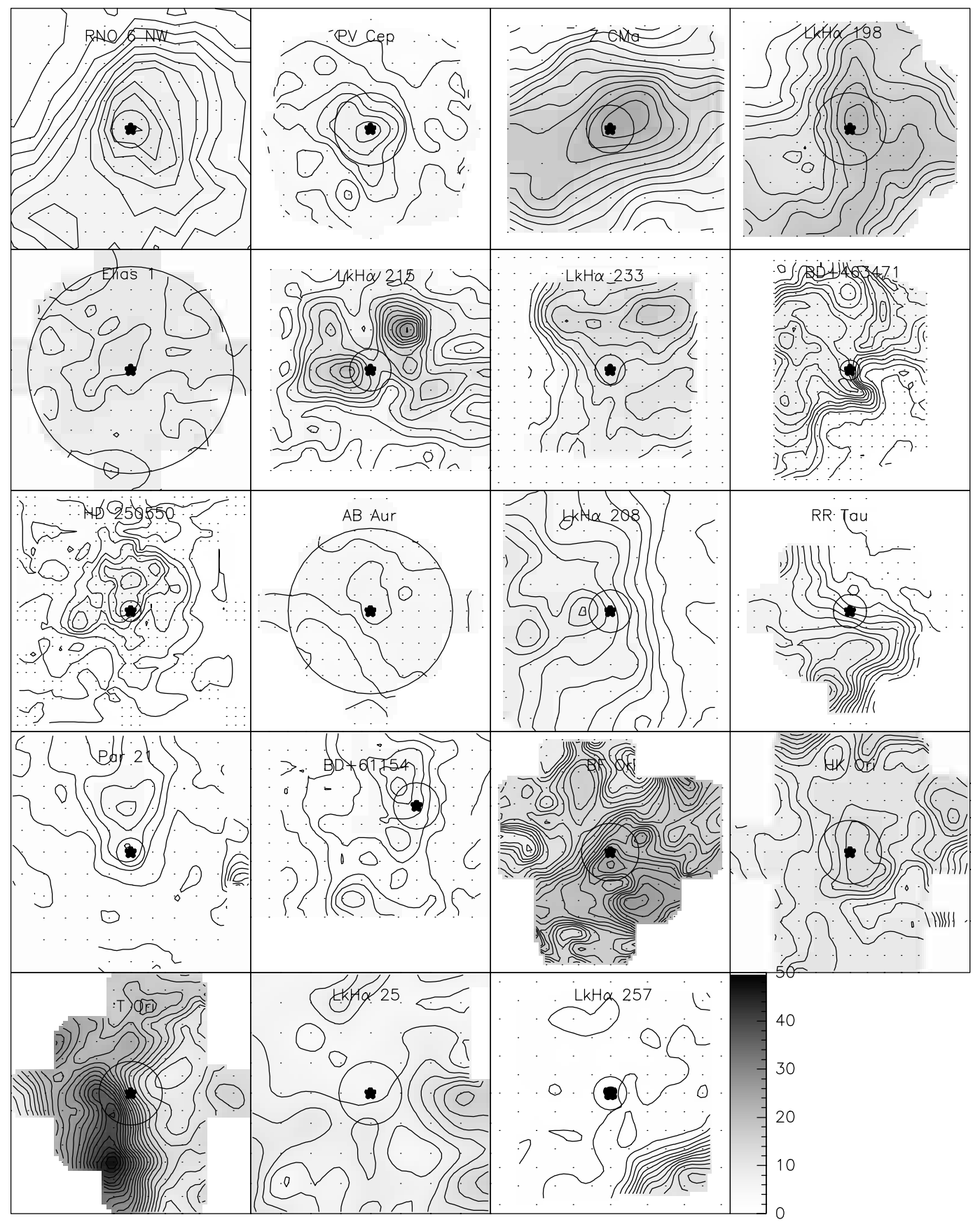

Fig. 2. The same as Fig. 1 for the late-type HAEBE stars of our sample. The size of the boxes varies from one source to the other. In each panel, a circle of $0.08 \mathrm{pc}$ of radius is drawn around the star. Contour levels are 0.3 to $70 \mathrm{by} 2 \mathrm{~K} \mathrm{~km} \mathrm{~s}^{-1}$. Like in Fig. 1 the stars are ordered in a tentative evolutionary sequence.

have been computed assuming a dust temperature of $30 \mathrm{~K}$ and an emissivity $\kappa_{1.3 \mathrm{~mm}}=0.015 \mathrm{~cm}^{2} \mathrm{gr}^{-1}$ (see Paper I). Because of the large uncertainty in the adopted value of the dust emissivity, these masses are uncertain by a factor of 4 .

Overall, we find that the values of the mass estimated from ${ }^{13} \mathrm{CO}, \mathrm{C}^{18} \mathrm{O}$ and the dust continuum emission differ by less than a factor of three. In three objects, MWC 1080 , PV Cep and $\mathrm{LkH} \alpha$ 198, the mass derived from the dust continuum emission is significantly lower than that derived from the ${ }^{13} \mathrm{CO}$ data. This is very likely due to the different angular resolution of the observations $\left(H P B W \sim 12^{\prime \prime}\right.$ at $1.3 \mathrm{~mm}$ and $\sim 24^{\prime \prime}$ at $3 \mathrm{~mm}$ ) and the chopping technique used in the continuum observations which could miss some extended emission.

\subsection{Spatial index $\alpha$}

In order to describe the morphology of the cloud around the star, we have defined the parameter $\alpha$ (called "spatial index" hereafter). This quantity is obtained by fitting the variation of the column density, $N_{T}$, as a function of 


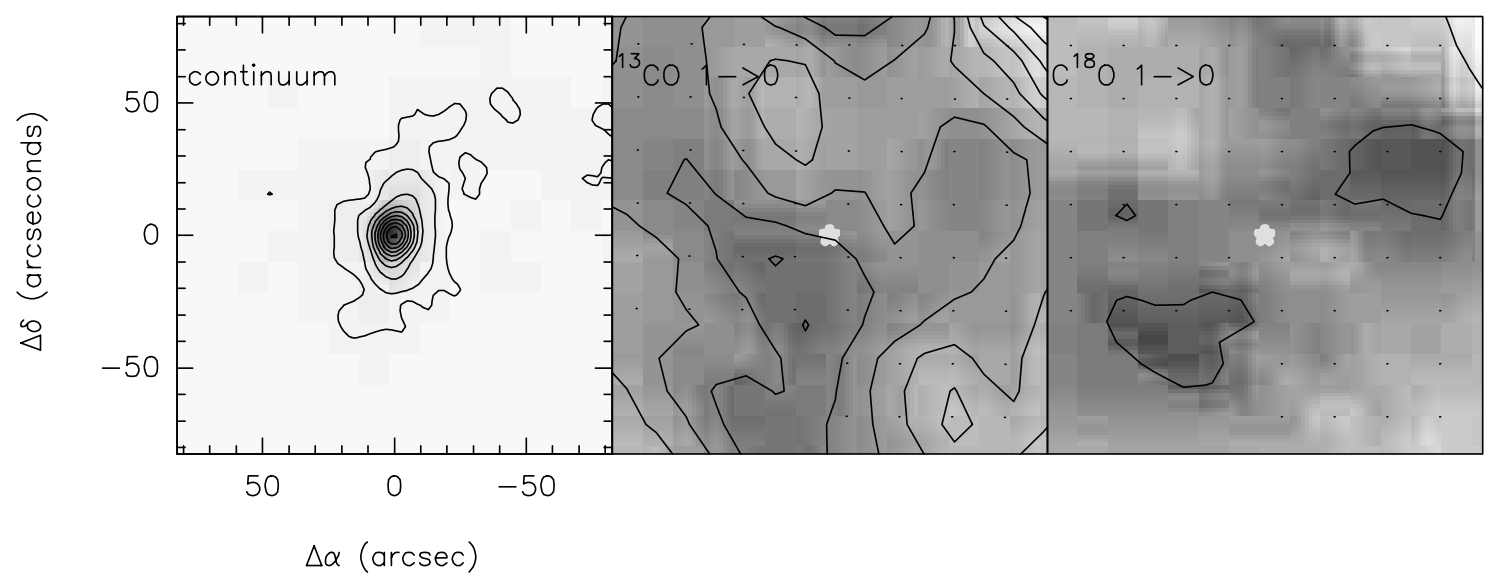

Fig. 3. a) Map of the dust continuum emission at $1.3 \mathrm{~mm}$ (right panel) toward the intermediate-mass protostar NGC 7129 FIRS 2. Contour levels are $40 \mathrm{mJy} /$ beam, $80 \mathrm{mJy} /$ beam to $728 \mathrm{mJy} /$ beam by $80 \mathrm{mJy} /$ beam; b) Integrated intensity map of the ${ }^{13} \mathrm{CO} 1 \rightarrow 0$ line towards the same region. Contour levels are 0.3 to 70 by $3 \mathrm{~K} \mathrm{kms}^{-1}$. c) The same for the $\mathrm{C}^{18} \mathrm{O} 1 \rightarrow 0$ line.

the distance from the star with a power law $N_{T} \propto r^{\alpha}$. Assuming spherical symmetry and a density law $n \propto r^{\beta}$, $\alpha$ is then related to $\beta$ by $\alpha=\beta+1$. Obviously, $\alpha$ depends on the spatial resolution of the observations, i.e., the distance of the star.

In order to have a uniform spatial scale in all the sources, we have calculated $\alpha$ by fitting the mean hydrogen column density in annular regions of 0.08 pc of thickness centered on the star. Since we have changed from an angular to linear scale, the number of observed positions within each annular region is dependent on the distance from the star. For early-type stars, we have fitted the column density profile up to a radius of $0.40 \mathrm{pc}$ from the star. For late-type stars, our maps do not cover such a large area, and we have used the whole mapped region (see Fig. 2). The small size of the regions mapped towards Elias 1 and $\mathrm{AB}$ Aur does not allow us to derive a value of $\alpha$. The mean hydrogen column density in each annulus has been estimated assuming uniform physical conditions. Under this approximation, the gas column density profile is proportional to the profile of the integrated line intensity, and the dust column density profile is proportional to the profile of the $1.3 \mathrm{~mm}$ continuum flux.

The integrated intensity profiles of the ${ }^{13} \mathrm{CO} 1 \rightarrow 0$ lines are shown in Figs. 4 and 5. In order to fit the profiles with a $N_{T} \propto r^{\alpha}$ law, we have linearized the function to $\log \left(N_{T}\right)=$ cte $+\alpha \log (r)$ and fitted the resulting straight line weighting each data point by $\sigma=\sigma_{N} / N_{T}$ where $\sigma_{N}$ is the rms of the map in column density units. In Paper I, we assumed equal weight for all data points in the linear regression fit which underemphasizes the uncertainties for small values of $N_{T}$. With the assumed weights, the uncertainties of all data points are assumed to be equal in the logarithmic scale (see Bevington 1969). The newly calculated values of $\alpha$ are given in Tables 3 and 4. Although small differences exist between the two estimates of $\alpha$, they do not significantly affect the results. In fact, as explained below, the differences between the values of $\alpha$ derived from different tracers are larger than those due to different weights.

The values of $\alpha$ obtained from ${ }^{13} \mathrm{CO}, \mathrm{C}^{18} \mathrm{O}$ and $1.3 \mathrm{~mm}$ continuum emission are shown in Tables 3 and 4 . We obtained different values of $\alpha$ depending on the mass tracer. In young objects using ${ }^{13} \mathrm{CO} \alpha \sim-0.5$, while $\alpha<-1$ from the dust continuum emission. Several factors can contribute to this difference. First of all, the opacity effects in the ${ }^{13} \mathrm{CO}$ line would produce a shallow distribution of the integrated line emission, and in our simple analysis, a lower absolute value of $\alpha$. This interpretation can explain those cases in which the value of $\alpha$ derived from the rarer isotope $\mathrm{C}^{18} \mathrm{O}$ is lower than that derived from ${ }^{13} \mathrm{CO}$ (RNO 6-NW, PV Cep and Z CMa). The values of $\alpha$ found from the $1.3 \mathrm{~mm}$ continuum data in young objects $(\alpha \sim-1.5$ to -2$)$ are smaller than those obtained from $\mathrm{C}^{18} \mathrm{O}(\alpha \sim-1$ to -0.5$)$. This is very likely due to the different angular resolution of the observations ( $H P B W \sim 12^{\prime \prime}$ at $1.3 \mathrm{~mm}$ and $\sim 24^{\prime \prime}$ at $3 \mathrm{~mm}$ ). The chopping thechnique used in the bolometer observations which could miss part of the more extended emission could also contribute to this difference. In the case of the young protostar NGC 7129-FIRS 2, optical depth effects could be important even in the $\mathrm{C}^{18} \mathrm{O}$ line.

We have plotted in Fig. 6 the values of $\alpha$ derived from the dust continuum emission against those derived from the ${ }^{13} \mathrm{CO}$ data. In all cases there is a general trend with the values of $\alpha$ derived from the dust continuum emission being lower than those derived from ${ }^{13} \mathrm{CO}$ data. However, for Type II and Type III stars the points lies almost in the diagonal of the plot, i.e., values of $\alpha$ from dust continuum and ${ }^{13} \mathrm{CO}$ emission are almost coincident. On the contrary, for protostars and Type I stars the absolute value of the difference between the values of $\alpha$ derived from $1.3 \mathrm{~mm}$ continuum emission and those from ${ }^{13} \mathrm{CO}$ data is $\gtrsim 0.5$. Besides the different angular resolution of 


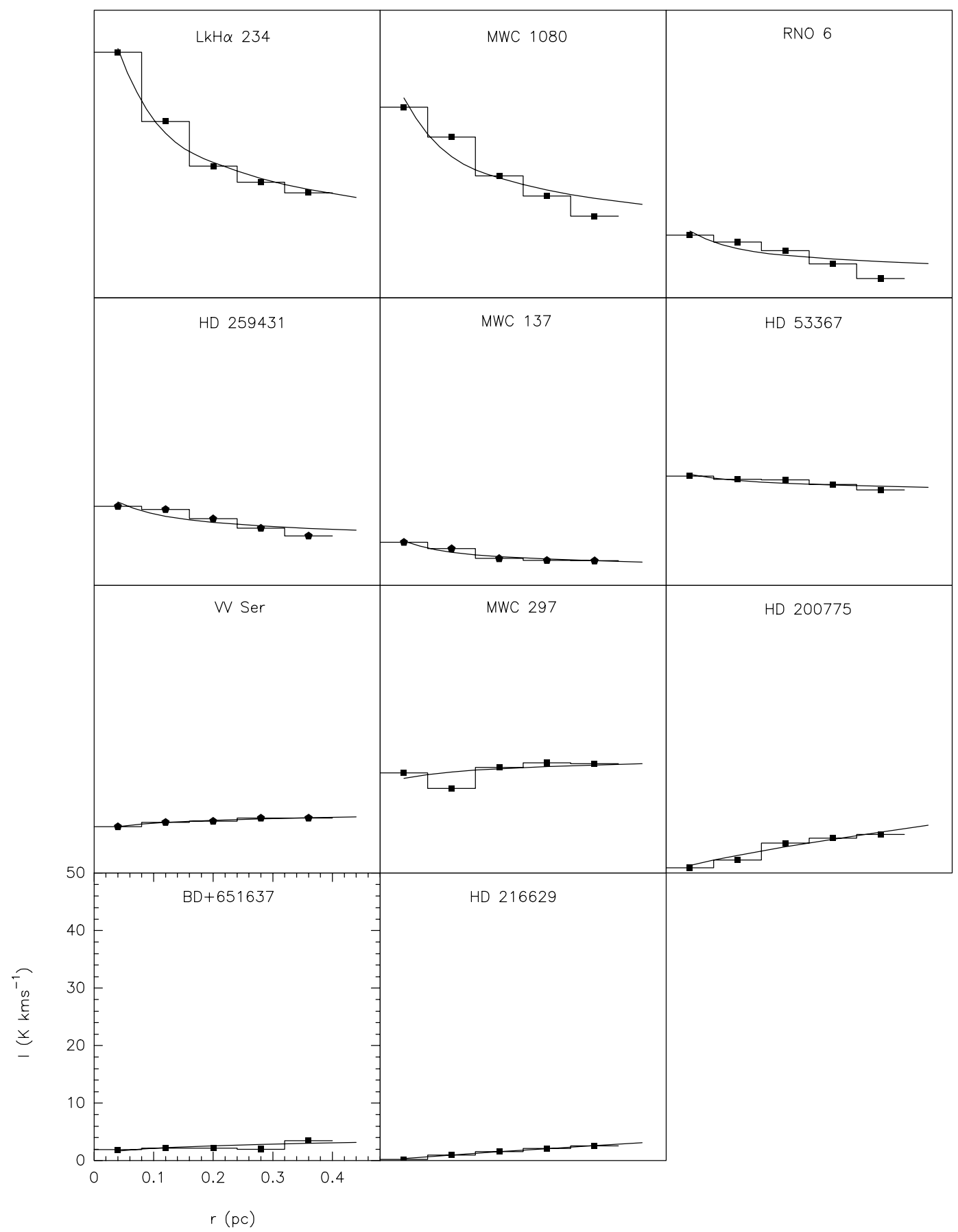

Fig. 4. Integrated intensity profiles around the early-type HAEBE stars. Each bin corresponds to an annular region with a thickness of $0.08 \mathrm{pc}\left(r_{\mathrm{out}}-r_{\mathrm{in}}=0.08 \mathrm{pc}\right)$. The fits $I \propto r^{\alpha}$ are also shown, and the values of $\alpha$ are listed in Table 3 .

the observations, opacity effects and molecular depletion contribute to explain this effect.

\subsection{Classifying HAEBE stars}

In Tables 3 and 4 we list the circumstellar mass and $\alpha$ values for all the stars in our sample. For clarity, we have ordered the stars following a tentative evolutionary sequence in which two general trends are clearly observed: i) the circumstellar mass decreases from $>10 M_{\odot}$ in the youngest objects to $<1 M_{\odot}$ in the more evolved stars; ii) the value of $\alpha$ increases from negative values in the youngest objects to positive values in the evolved stars. These trends are independent of the mass tracer used in the calculations. According to this evolutionary sequence and following the method described in Paper I, we classify HAEBE stars in three groups: Type I stars have $-2<\alpha \leq-1$; Type II stars have $-1<\alpha \leq 0$; and in Type III stars $\alpha>0$.

However, since the value of $\alpha$ depends somewhat on the mass tracer, we must identify the most suitable tracer for each Type. For protostars and Type I stars, we adopt the circumstellar masses and $\alpha$ values derived from the $1.3 \mathrm{~mm}$ 


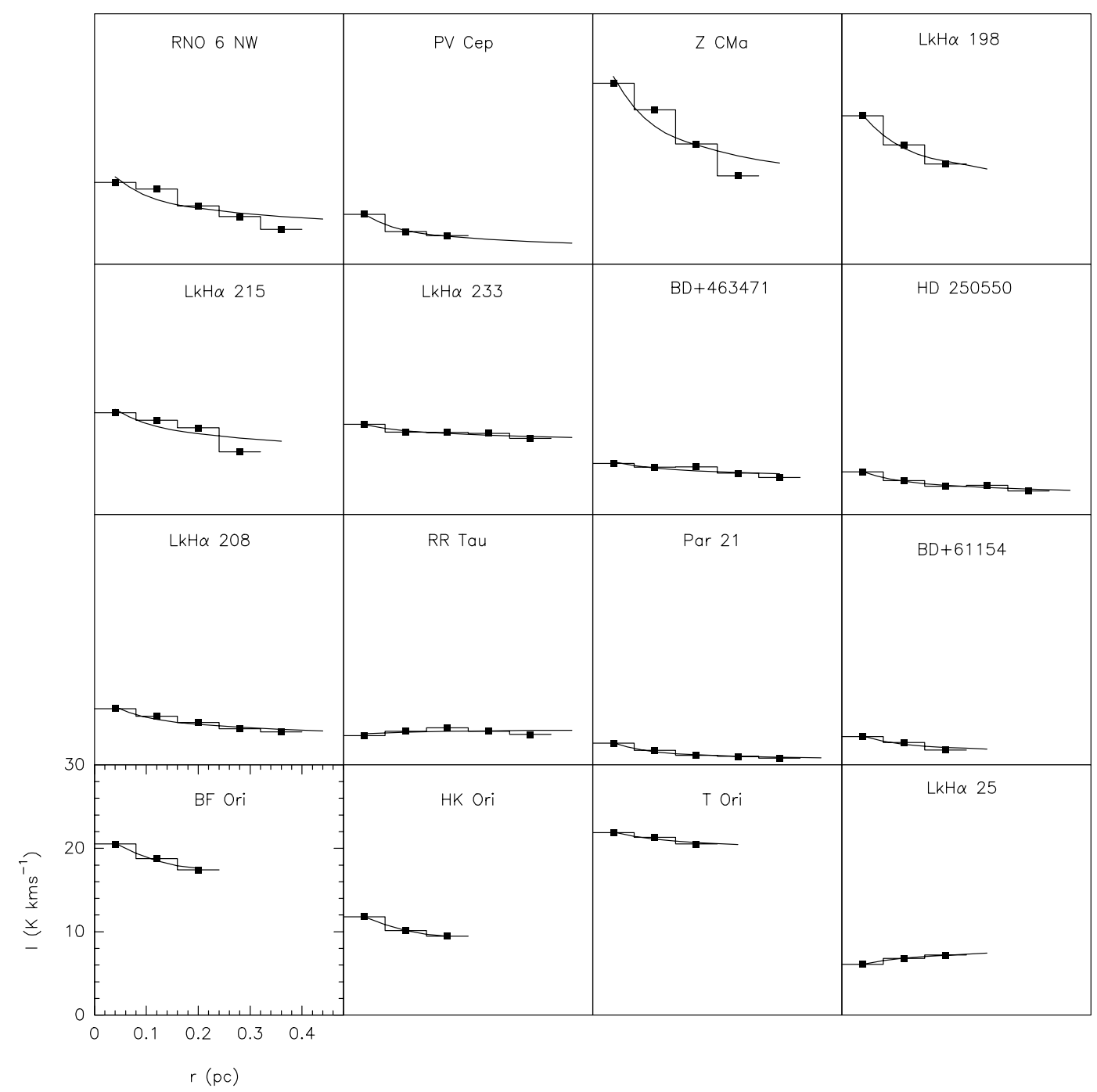

Fig. 5. Same as Fig. 4 but for late-type stars. The fits $I \propto r^{\alpha}$ are also shown, and the values of $\alpha$ are listed in Table 4 .

continuum emission. In the case of RNO 6-NW we do not have a continuum image, and we take the value of $\alpha$ derived from $\mathrm{C}^{18} \mathrm{O}$ which is, in fact, an upper limit. Continuum emission towards PV Cep was only detected in a clump of size less than $0.08 \mathrm{pc}$, too small to allow a fit of $\alpha$, and we use the value provided by $\mathrm{C}^{18} \mathrm{O}$. Finally, for Type II and III stars we use the ${ }^{13} \mathrm{CO}$ data to compute masses and $\alpha$ values. In Tables 3 and 4 we have marked in boldface the values of the circumstellar mass and $\alpha$ used to classify the HAEBE star and in the discussion of the following sections.

\section{Mass dispersal in intermediate-mass objects}

In order to have a comprehensive view of the process of mass dispersal during the entire evolution of Herbig stars, we have observed 2 and compiled data on 15 intermediatemass protostars. In Table 2 we show the circumstellar masses adopted from the literature which are, when possible, those derived from the dust continuum emission (see references in Table 2). For some sources (IRAS 20126,
L1455M-FIR, G192.16, GGD27-IRS) we have found a large dispersion in the values of the circumstellar masses. Since the most important observational effects such as missed flux in interferometric observations and large opacity in molecular lines tend to underestimate the actual value of the circumstellar mass, we have adopted the largest one.

The circumstellar masses and $\alpha$ values as a function of the luminosity for the protostars (circles) and the three types of Herbig stars (Type I: squares, Type II: triangles, Type III: stars) are shown in Fig. 7. In the case of protostars, we have been able to derive the value of $\alpha$ only for those observed by us. In spite of the non-uniformity of our sample of protostars, Fig. 7 clearly shows a trend in which the amount of circumstellar material around protostars is correlated with the bolometric luminosity, varying from a few solar masses around the low luminosity sources to $\sim 400 M_{\odot}$ for the brightest objects. A similar trend was observed by Brand et al. (2001) in a more uniform sample of high-mass protostellar candidates, suggesting that the correlation between the mass of the parent clump and 
Table 3. Observational results.

\begin{tabular}{|c|c|c|c|c|c|c|c|c|c|c|}
\hline \multicolumn{11}{|c|}{ Spectral types B0-B5 } \\
\hline \multirow[t]{2}{*}{ Object } & \multicolumn{3}{|c|}{ Morphology $^{1}$} & \multicolumn{3}{|c|}{$\operatorname{Mass}_{R=0.08 \mathrm{pc}}\left(M_{\odot}\right)^{2}$} & \multicolumn{3}{|c|}{$\alpha^{3}$} & \multirow[t]{2}{*}{ Type } \\
\hline & ${ }^{13} \mathrm{CO}$ & $\mathrm{C}^{18} \mathrm{O}$ & $1.3 \mathrm{~mm}$ & ${ }^{13} \mathrm{CO}$ & $\mathrm{C}^{18} \mathrm{O}$ & $1.3 \mathrm{~mm}$ & ${ }^{13} \mathrm{CO}$ & $\mathrm{C}^{18} \mathrm{O}$ & $1.3 \mathrm{~mm}$ & \\
\hline N-FIRS 2 & Off-peak & Off-peak & Peak & 7.7 & 9.5 & 15.0 & -0.0 & -0.1 & -1.4 & \\
\hline $\mathrm{LkH} \alpha 234$ & Peak & Peak & Peak & 13.8 & 14.7 & 16.6 & -0.4 & -0.4 & -1.2 & I \\
\hline MWC 1080 & Peak & Off-P & Off-P & 10.8 & 7.5 & 3.3 & -0.3 & -0.03 & -0.64 & II \\
\hline RNO 6 & Off-P & Off-P & & 3.5 & 2.5 & & -0.3 & -0.3 & & II \\
\hline HD 259431 & Off-P & Off-P & & 4.5 & 5.0 & & -0.2 & -0.1 & & II \\
\hline MWC 137 & Off-P & Off-P & Off-P & 2.4 & $<0.6$ & 1.4 & -0.3 & & -0.3 & II \\
\hline HD 53367 & Off-P & Off-P & & 6.1 & 7.2 & & -0.05 & -0.2 & & II \\
\hline VV Ser & Cavity & Cavity & & 2.6 & 2.3 & & 0.1 & 0.0 & & III \\
\hline MWC 297 & Cavity & Cavity & & 5.6 & 3.6 & & 0.1 & 0.2 & & III \\
\hline HD 200775 & Cavity & & Cavity & 0.6 & $<0.6$ & & 0.8 & & & III \\
\hline $\mathrm{BD}+651637$ & Cavity & & Cavity & 0.6 & $<0.4$ & 0.5 & 0.3 & & 0.0 & III \\
\hline HD 216629 & Cavity & Cavity & & 0.10 & $<0.4$ & & 0.9 & & & III \\
\hline BНJ 71 & Cavity & No & & $\leq \mathbf{0 . 0 4}$ & & & & & & III \\
\hline HD 37490 & No & No & & $\leq \mathbf{0 . 0 2}$ & & & & & & III \\
\hline HD 52721 & No & No & & $\leq \mathbf{0 . 0 6}$ & & & & & & III \\
\hline
\end{tabular}

${ }^{1}$ Brief description of the morphology of the cloud in ${ }^{13} \mathrm{CO}, \mathrm{CS}$ and $1.3 \mathrm{~mm}$ continuum emission. Peak: the HAEBE star is located at the emission peak; Off-P: the star is located off by more than a HPBW from the star; Cavity: the star is located in a cavity; No: undetected.

${ }^{2}$ Mass in a radius of $0.08 \mathrm{pc}$ around the star estimated from ${ }^{13} \mathrm{CO}, \mathrm{C}^{18} \mathrm{O}$ and $1.3 \mathrm{~mm}$ continuum emission.

${ }^{3}$ Exponent of the column density law $N_{m} \propto r^{\alpha}$ estimated from ${ }^{13} \mathrm{CO}, \mathrm{C}^{18} \mathrm{O}$ and $1.3 \mathrm{~mm}$ continuum maps.

Note: Bold numbers in Tables 3 and 4 indicate the values of the circumstellar masses and $\alpha$ used for the classification of HAEBE stars.

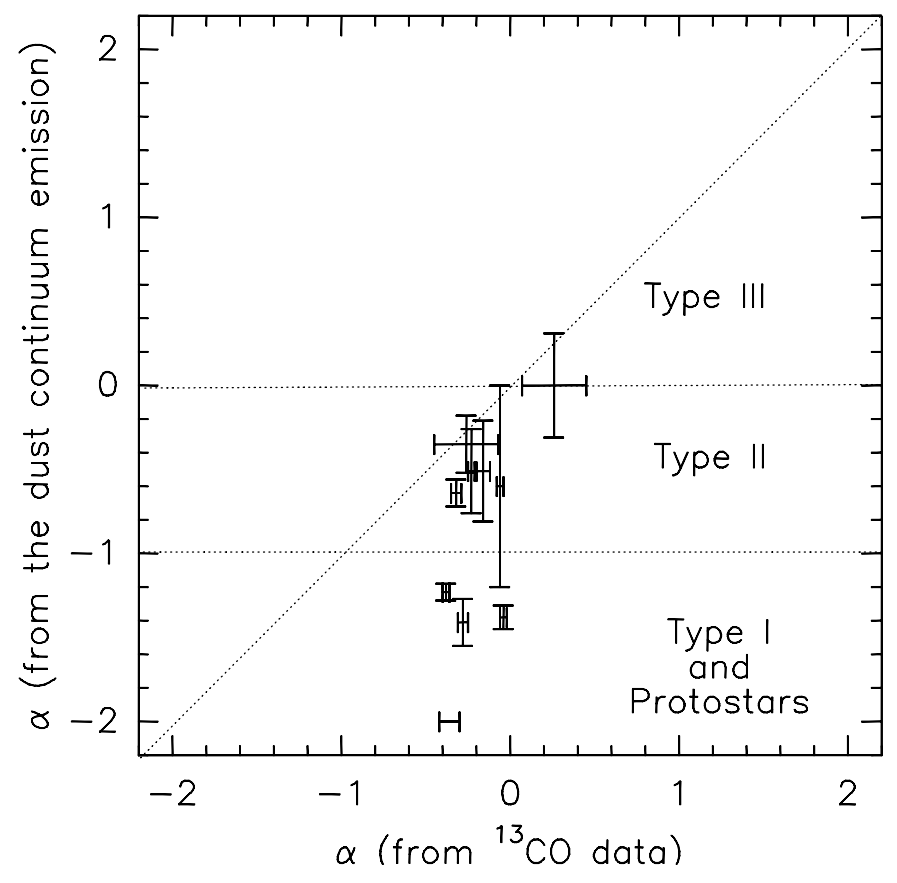

Fig. 6. Spatial index $\alpha$ derived from continuum data against $\alpha$ derived from ${ }^{13} \mathrm{CO}$ data. Note that both values are coincident (within the error bars) for Type II stars. However, $\alpha$ from continuum data is lower than $\alpha$ from ${ }^{13} \mathrm{CO}$ data in Type I stars and protostars.

the bolometric luminosity in protostars extends over a wide range of luminosities $\left(L_{\text {bol }}\right.$ from $\sim 10$ to $\left.10^{5} L_{\odot}\right)$.
Since we have observed only 3 Type I stars, we cannot say much about their properties. The situation for Type II stars differs from that of protostars. We do not find a dependence of the circumstellar mass on stellar luminosity, with the mass varying in the narrow interval 1 to $10 M_{\odot}$ over the whole luminosity range (4 orders of magnitude). Finally, smaller circumstellar masses, $<1 M_{\odot}$, are found in Type III sources. These data are summarized in Table 5. Comparing the circumstellar masses in protostars with those towards Type I and II objects, we conclude that most of the circumstellar material $(>90 \%)$ is dispersed by the time the star becomes a Type I object. This is not surprising since the star must disperse a large fraction of the envelope to become visible. Since the circumstellar mass in protostars correlates with the bolometric luminosity, the amount of mass dispersed during this period is strongly dependent on this stellar parameter. Note also that protostars and Herbig stars of low luminosities $\left(<100 L_{\odot}\right)$ have similar amounts of circumstellar matter.

The values of $\alpha$ estimated for the two protostars, NGC 7129-FIRS 2 and RNO 6-NW, are similar to those of Type I stars $(\alpha \approx-1)$. Chandler \& Richer (2000) imaged the submillimeter dust continuum emission of eight young low- and intermediate-mass protostars and found that the density profiles can be fitted by a power law $n \sim r^{\beta}$, with $\beta \sim-1.5$ in Class 0 objects and $\beta \sim-2$ towards Class I sources (equivalent to $-1 \leq \alpha \leq-0.5$ ). These values are in agreement with our results for NGC 7129-FIRS 2 and RNO 6-NW and suggest that protostars are well described 
Table 4. Observational results.

\begin{tabular}{|c|c|c|c|c|c|c|c|c|c|c|}
\hline \multicolumn{11}{|c|}{ Spectral types later than B6 } \\
\hline \multirow[t]{2}{*}{ Object } & \multicolumn{3}{|c|}{ Morphology $^{1}$} & \multicolumn{3}{|c|}{$\operatorname{Mass}_{r=0.08 \mathrm{pc}}\left(M_{\odot}\right)^{2}$} & \multicolumn{3}{|c|}{$\alpha^{3}$} & \multirow[t]{2}{*}{ Type } \\
\hline & ${ }^{13} \mathrm{CO}$ & $\mathrm{C}^{18} \mathrm{O}$ & $1.3 \mathrm{~mm}$ & ${ }^{13} \mathrm{CO}$ & $\mathrm{C}^{18} \mathrm{O}$ & $1.3 \mathrm{~mm}$ & ${ }^{13} \mathrm{CO}$ & $\mathrm{C}^{18} \mathrm{O}$ & $1.3 \mathrm{~mm}$ & \\
\hline RNO 6-NW & Peak & Peak & & 3.2 & 5.2 & & -0.3 & -0.5 & & \\
\hline PV Cep & Peak & Peak & Peak & 1.9 & 4.1 & 1.0 & -0.4 & -0.7 & $-2.0^{4}$ & $\mathrm{I}$ \\
\hline $\mathrm{Z} \mathrm{CMa}$ & Peak & Peak & Peak & 7.0 & 10.8 & 6.6 & -0.3 & -0.5 & -1.4 & I \\
\hline $\mathrm{LkH} \alpha 198$ & Peak & Off-P & Off-P & 5.8 & 9.7 & 2.3 & -0.2 & -0.7 & -0.5 & II \\
\hline Elias 1 & Peak & Off-P & & 2.9 & 7.8 & & & & & II \\
\hline $\mathrm{LkH} \alpha 215$ & Off-P & Cavity & Off-P & 3.9 & 1.6 & 0.9 & -0.2 & 0.2 & -0.5 & II \\
\hline $\mathrm{LkH} \alpha 233$ & Off-P & Off-P & Off-P & 3.5 & 7.2 & 0.5 & -0.1 & -0.3 & -0.6 & II \\
\hline $\mathrm{BD}+463471$ & Edge & Cavity & & 2.0 & 1.9 & & -0.1 & -0.1 & & II \\
\hline HD 250550 & Off-P & Off-P & & 1.6 & 0.7 & & -0.2 & 0.0 & & II \\
\hline AB Aur & Peak & Edge & & 1.3 & 1.1 & & & & & II \\
\hline $\mathrm{LkH} \alpha 208$ & Edge & Cavity & & 1.6 & $<1$ & & -0.2 & $>0$ & & II \\
\hline RR Tau & Edge & Cavity & & 1.1 & $<0.4$ & & 0.0 & & & II \\
\hline Par 21 & Peak & Cavity & No & 0.85 & 0.65 & & -0.5 & & & II \\
\hline $\mathrm{BD}+61154$ & Off-P & No & & 1.1 & $\leq 0.5$ & & -0.3 & & & II \\
\hline BF Ori & Peak & Off-P & & 6.1 & 3.4 & & -0.1 & 0.1 & & II \\
\hline HK Ori & Peak & Cavity & No & 3.4 & 2.6 & $<0.5$ & -0.1 & 0.1 & & II \\
\hline T Ori & Edge & Cavity & & 7.1 & 3.2 & & 0.0 & 0.0 & & II \\
\hline $\mathrm{LkH} \alpha 25$ & Cavity & No & No & 2.0 & $\leq 0.8$ & & 0.1 & & & III \\
\hline $\mathrm{LkH} \alpha 218$ & No & No & & $<0.02$ & & & & & & III \\
\hline $\mathrm{LkH} \alpha 257$ & No & No & & $\sim 0.10$ & & & $>0$ & & & III \\
\hline HD 245185 & No & No & & $<\mathbf{0 . 0 2}$ & & & & & & III \\
\hline
\end{tabular}

${ }^{1}$ Brief description of the morphology of the cloud in ${ }^{13} \mathrm{CO}, \mathrm{CS}$ and $1.3 \mathrm{~mm}$ continuum emission. Peak: the HAEBE star is located at the emission peak; Off-P: the star is located off by more than a HPBW from the star; Cavity: the star is located in a cavity; No: undetected.

${ }^{2}$ Mass in a radius of $0.08 \mathrm{pc}$ around the star estimated from ${ }^{13} \mathrm{CO}, \mathrm{C}^{18} \mathrm{O}$ and $1.3 \mathrm{~mm}$ continuum emission.

${ }^{3}$ Exponent of the column density law $N_{m} \propto r^{\alpha}$ estimated from ${ }^{13} \mathrm{CO}, \mathrm{C}^{18} \mathrm{O}$ and $1.3 \mathrm{~mm}$ continuum maps.

${ }^{4}$ The radius of the $1.3 \mathrm{~mm}$ clump is $<0.08 \mathrm{pc}$. For this reason, it is classified as a Type I star, although in the figures we plot a value of $\alpha$ of -0.7 .

Note: Bold numbers in Tables 3 and 4 indicate the values of the circumstellar masses and $\alpha$ used for the classification of HAEBE stars.

by a density law $n \sim r^{\beta}$ and $-2<\beta<-1.5$, implying that the value of $\alpha$ does not increase, in fact it seems to decrease, from protostars to Type I objects (see Fig. 7 and Table 5).

The situation changes substantially when one compares Type I, Type II and Type III stars. Along this sequence, $\alpha$ increases continuously from -1 to $>0$ due to the dramatic change of the morphology of the cloud from a centrally peaked clump to a large cavity in the parent molecular cloud. A very weak dependence of $\alpha$ on the stellar luminosity appears to exist in Type I and II stars (see Fig. 7). The situation is somewhat different for Type III stars. High luminosity sources (early-type HAEBE stars) reach values of $\alpha$ well above zero, while in late-type HAEBE stars $\alpha \sim 0$. This indicates that only early-type HAEBE stars are able to excavate cavities in the molecular cloud during their pre-main-sequence (PMS) evolution (see also Fig. 2). This difference in the efficiency of gas and dust removal between early- and latetype stars is clearly seen in Fig. 8. This figure shows the frequency distribution of $\alpha$ in these stars. All the stars with $\alpha>0.5$ and most with $\alpha>0$ are early-type stars. Late-type stars reach the main-sequence (MS) with $\alpha$ between -0.5 and 0 .

\subsection{Mass dispersal mechanisms from protostars to Type I HAEBE stars}

This is the phase during which most of the circumstellar envelope is dispersed: around $90 \%$ of the mass of the parent clump. Assuming that the bolometric luminosity in protostars represents the stellar luminosity (and not due to accretion), we find that early-type stars vary from circumstellar masses between $30-400 M_{\odot}$ in the protostellar phase to less than or about $10 M_{\odot}$ when they become a Type I/II Herbig star. Late-type stars go from about 3-80 $M_{\odot}$ in the protostellar phase to about $1-10 M_{\odot}$ as Type I/II star. However, the spatial index $\alpha$ remains nearly constant $(\alpha \sim-1)$. Bipolar outflows appear to be the unique efficient mechanism that can explain this effect. 

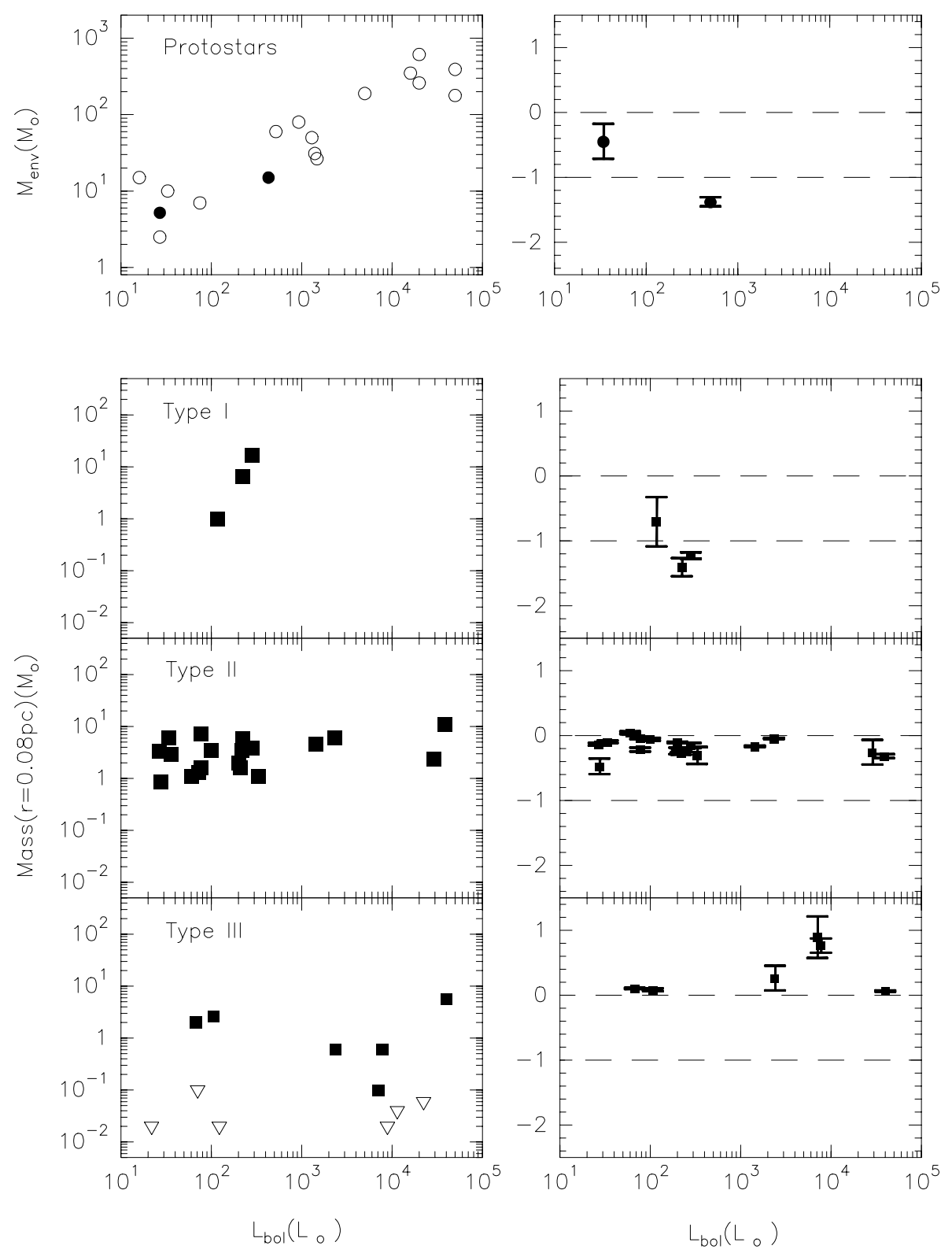

Fig. 7. The circumstellar mass and the spatial index $\alpha$ vs. bolometric luminosity for protostars (filled circles are NGC 7129FIRS 2 and RNO 6 - NW and empty circles are the protostars listed in Table 2), Type I, Type II, and Type III HAEBE stars. Inverted open triangles are upper limits of the circumstellar mass.

Bipolar outflows sweep out the material along the poles and create a biconical cavity. Material in the envelope falls to feed the growth of the circumstellar disk and eventually the star. In this way, the cloud evolves towards a more centrally peak morphology while a significant fraction of the core material is dispersed.

On the other hand, bipolar outflows can explain the larger mass dispersal in early-type stars. It is well known that accretion and mass-loss activity are closely related. Several studies have attempted to find a correlation between the flow energetics and the bolometric luminosity of the driving source. For example, Cabrit \& Bertout (1992) found that both the luminosity and the momentum flux of the outflow are correlated with the bolometric luminosity $\left(L_{\mathrm{CO}} \propto L_{\mathrm{bol}}^{0.8}\right)$. The data shown in Fig. 7 and previous studies on protostars Brand et al. (2001) suggest that the amount of circumstellar mass is also correlated with the bolometric luminosity, and therefore, with the mechanical luminosity and momentum flux of the outflow. Cabrit \& André (1991) suggested a similar correlation between the mass of the circumstellar disk and the outflow energetics based on a sample of low-mass protostars. If the outflow luminosity and momentum flux are correlated with the circumstellar mass, it is not surprising that the outflow disperses a large fraction of the core even in the more luminous/massive sources.

We propose that bipolar outflows are the main mass dispersal mechanism at the first stages $(<0.1 \mathrm{Myr})$ of the evolution of early-type and late-type intermediate mass stars. An important question is whether the star formation 
Table 5. Circumstellar material.

\begin{tabular}{lcc}
\hline \hline & $\begin{array}{c}\operatorname{Mass}_{R=0.08 \mathrm{pc}} \\
\left(M_{\odot}\right)\end{array}$ & $\alpha$ \\
\hline Protostars & $10-500$ & $\sim-1$ \\
Type I & $1-20$ & $\leq-1$ \\
Type II & $1-10$ & -1 to 0 \\
Type III & $\lesssim 1$ & $>0$ \\
\hline
\end{tabular}

efficiency, i.e., the fraction of the envelope mass which eventually becomes a star, is similar in early- and latetype stars. The fact that there is no correlation between the circumstellar mass and the luminosity of the sources in Type II stars suggests that this is not the case. It seems that early-type stars disperse a larger fraction of the envelope mass than late-type stars. This result has important consequences for our understanding of the star formation process itself. Recent studies in $\rho$ Oph (Motte et al. 1998) and in Serpens (Testi et al. 2000) show that the mass distribution of the clumps in molecular clouds is similar to the initial mass function (IMF) of field stars, suggesting that the latter is already determined by the fragmentation of the molecular cloud. This implies that the star formation efficiency is the same across the entire mass range, a result at odds with our findings. We recall that our observations are restricted to HAEBE stars and do not account for the early stages of the star formation process. On the other hand, $\rho \mathrm{Oph}$ and Serpens regions are forming low-mass and late-type intermediate mass stars. Similar studies of the IMF in molecular clouds with highmass star formation are needed in order to verify whether the efficiency of star formation decreases with increasing mass.

The importance of bipolar outflows in the the mass dispersal around low-mass stars has been pointed out by several authors (see e.g. Myers et al. 1988; Ladd et al. 1998). Velusamy \& Langer (1998) presented high-angular resolution observations of the outflow/infall interaction towards the low-mass star IRS1 in Barnard 5. They observed a wider opening angle of the outflow near the star of $125^{\circ}$ and suggested that if the widening of the outflow continues, the disk will be isolated from the core and the infall will stop. We propose that the bipolar outflow is also an important mass dispersal agent in the first evolutionary stages of intermediate-mass stars. Detailed studies of the relationship between infall and outflow in these objects are required to understand the processes which determine the star formation efficiency.

\subsection{Mass dispersal from Type I to Type III HAEBE stars: "Early-type" vs. "Late-type" stars}

The process of mass dispersal in HAEBE stars has a very different characteristic than in protostars. The morphology of the cloud completely changes during this epoch: $\alpha$ increases from $\sim-1$ in Type I stars to positive values in Type III stars. However, the amount of mass dispersed

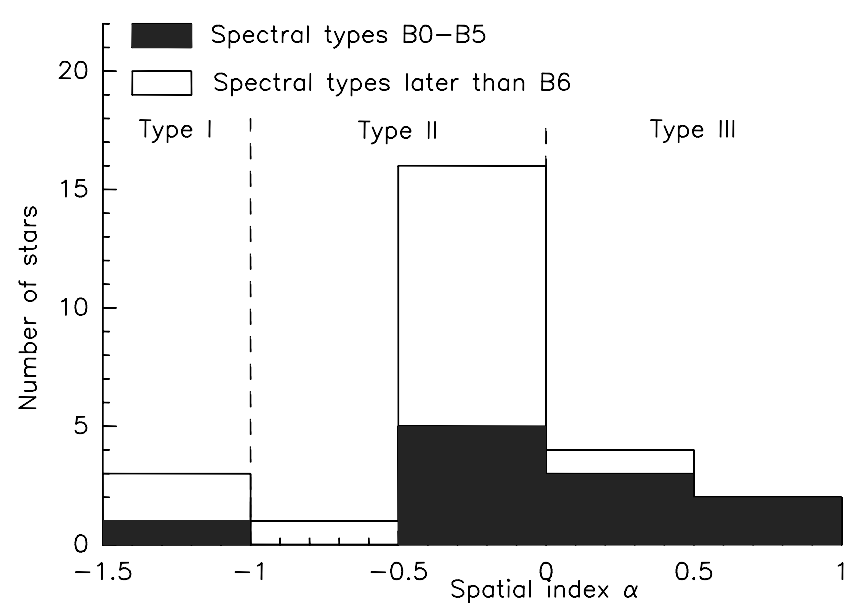

Fig. 8. Histogram of the frequency distribution of the $\alpha$ values for early-type (B0-B5) and late-type (later than B6) stars. Only stars with calculated values of $\alpha$ are included. Dashed vertical lines separate Type I, II and III stars.

during this phase (of the order of tenths of $M_{\odot}$ ) is modest compared to the amount of mass dispersed during the protostellar phase. Different mechanisms should account for this process in this evolutionary stage. In order to investigate the characteristic time-scale, we have plotted in Fig. 9 the circumstellar mass and $\alpha$ as a function of stellar age for the early- and late-type stars (those for which we have a reliable age estimate).

There seems to be a correlation between $\alpha$ and the stellar age for early-type stars: $\alpha$ changes from values $\sim-1$ for stars with ages $\sim 0.1$ Myr to values $>0$ in stars older than $\sim 1$ Myr. This suggests a close correlation between the morphology of the circumstellar material and the age of the star. A similar correlation between the stellar age and $\alpha$ is also found in late-type HAEBE stars, whereby $\alpha \sim-0.5$ in stars with ages $\sim 0.1 \mathrm{Myr}$ and $\alpha \sim 0$ for objects $\sim 1$ Myr old. However, $\alpha$ never reaches values as large $(\gg 0)$ as in early-type stars. In other words, these less massive stars seem to be unable to excavate a cavity in the molecular cloud during their pre-main-sequence (PMS) evolution, so that $\alpha$ takes a fairly constant value of 0 for the more evolved objects.

The correlation between the circumstellar mass and the stellar age is not as clear as in the case of $\alpha$. In earlytype stars, the circumstellar mass certainly decreases with the stellar age. However, the data points cannot be fitted with a single linear regression fit. On the contrary, there appear to be two different regimes: a quasi-stationary phase for ages between 0.1 and $1 \mathrm{Myr}$, followed by a rapid dispersal of the remnant material at later ages. The critical age seems to be $\sim 1 \mathrm{Myr}$, which is also the age at which $\alpha$ changes from negative to positive values. Based on these data we estimate a lifetime of $\sim 1 \mathrm{Myr}$ for the envelope of these massive stars.

In late-type stars, the correlation between the circumstellar mass and the stellar age is less clear. For ages between 0.1 and $1 \mathrm{Myr}$, the dispersion in the values of the circumstellar mass is of the same order than the absolute 

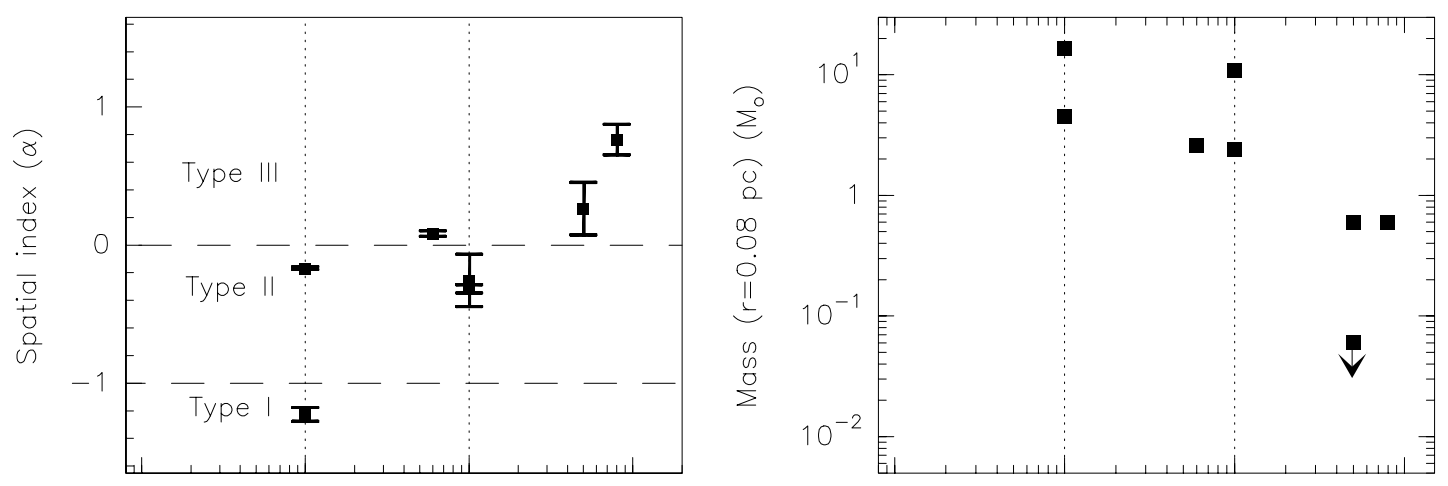

Spectral types later than B6
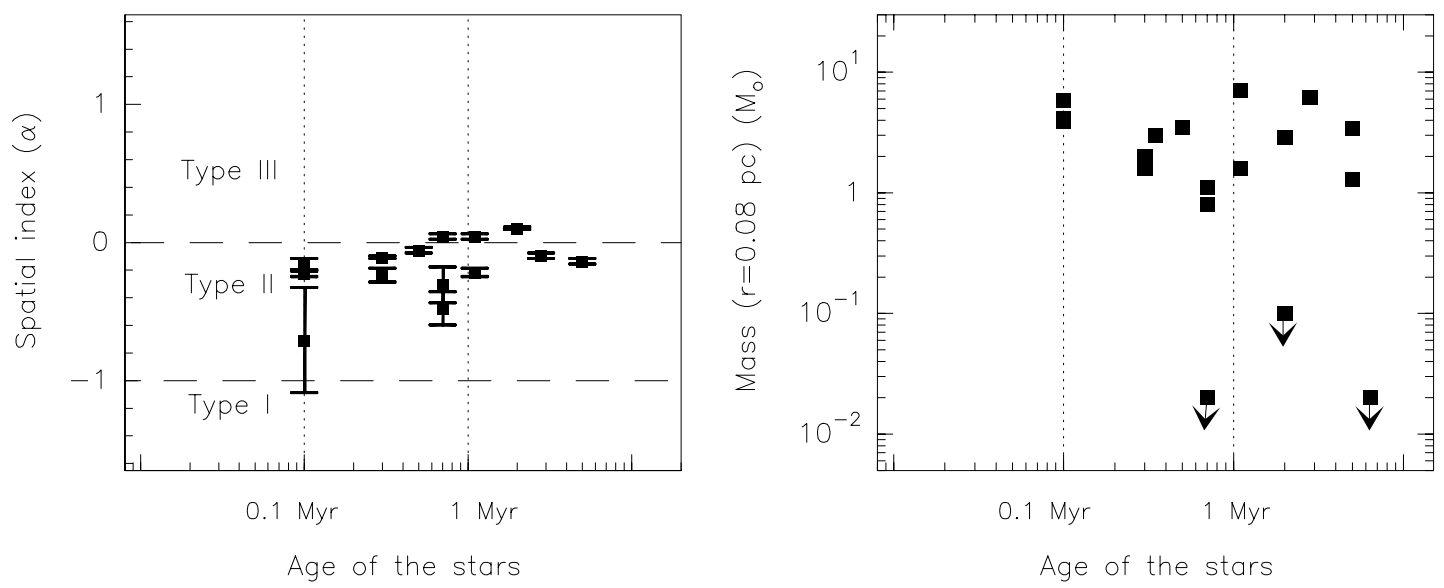

Fig. 9. Spatial index, $\alpha$, derived as explained in the text, and circumstellar mass vs stellar ages for early-type (top) and late-type (bottom) HAEBE stars.

value of the masses $\left(<10 M_{\odot}\right)$, and a correlation between circumstellar mass and the stellar age cannot be established. As commented in Sect. 4.1, our mass estimates include material along the line of sight which could not be physically associated with the star. The confusion with the foreground cloud can introduce large uncertainties in the estimates of the circumstellar masses of these evolved objects. Interferometric imaging and/or the observation of high excitation lines are necessary in order to determine which fraction of the mass is actually circumstellar mass and establish its actual dependence on the stellar age. Since $\alpha$ takes values of $\sim 0$ even for the older stars, we conclude that the lifetime of the envelope could be as large as $\sim 10 \mathrm{Myr}$ in these late-type stars. Finally, we note the less frequent case where some late-type objects with ages $>1$ Myr have completely dispersed the envelope.

Our previous discussion is based on the age estimates listed in Table 1. Naturally, age estimates are affected by uncertainties that result both from the use of a given set of evolutionary tracks and from errors in the stellar parameters. Of these two sources, the latter is the most serious one. In fact, Herbig Ae/Be stars are almost fully radiative objects whose exact location in the H-R diagram is not sensitive to the details of the input physics of the models, but mostly on the stellar mass. Thus, different theoretical tracks are in good agreement with each other. However, isochrones may differ substantially if one does not allow for a proper treatment of the initial conditions. We have adopted ages starting from the birthline which provides a more physical basis for the initial time of pre-mainsequence contraction.

On the other hand, the heterogenous nature of the ensemble of Herbig Ae/Be stars still precludes an accurate knowledge of both the effective temperature and luminosity of each member of the group. Differences in spectral types available in the literature can be large (up to 2-3 subclasses), while distances based on Hipparcos are known only for a small subset (e.g. van den Ancker 1998). Our choices of temperature and luminosity are based on a careful examination of various compilations (Hillenbrand et al. 1992; Berrilli et al. 1992; van den Ancker et al. 1998). Although there may be large differences in the stellar ages for individual cases, the general evolutionary trend discussed in this paper is not affected.

\subsection{Stellar winds and photodissociation as possible mass dispersal mechanisms in HAEBE stars}

Bipolar outflows are not a plausible mechanism for mass dispersal in HAEBE stars. First of all, the association of 
HAEBE stars with bipolar outflows is not clear. Very few energetic outflows have been detected towards HAEBE stars and in a few well studied cases of apparent association of a HAEBE star with an outflow, the outflow turned out to be driven by a deeply embedded infrared companion $(\mathrm{LkH} \alpha$ 198, Sandell et al. 1994; $\mathrm{LkH} \alpha$ 234, Fuente et al. 2001). Furthermore, since these outflows are strongly bipolar they cannot explain the morphology of the cavities observed in some early-type stars. Stellar winds and photodissociation seem more plausible agents in these objects.

HAEBE stars are believed to possess almost fully radiative interiors. Yet, in several cases strong ionized winds have been discovered. Skinner et al. (1993) carried out a survey at centimetre wavelengths and detected 12 stars out of a sample of 57 objects. Skinner et al. (1993) concluded that the derived spectral indices are consistent with those expected for a spherical constant velocity wind. The derived ionized mass-loss rates varied between $\sim 10^{-8} M_{\odot} \mathrm{yr}^{-1}$ for late-type stars and $\sim 10^{-7} M_{\odot} \mathrm{yr}^{-1}$ for early-type stars (Skinner 1994). Assuming a typical velocity for the ionized wind of $200 \mathrm{~km} \mathrm{~s}^{-1}$, early-type stars inject in the surrounding cloud a momentum flux of $2 \times 10^{-5} M_{\odot} \mathrm{yr}^{-1} \mathrm{~km} \mathrm{~s}^{-1}$. Typical CO profiles towards HAEBE stars present moderate wings with terminal velocities of $\approx 5 \mathrm{kms}^{-1}$. Assuming that this neutral gas is being driven by the ionized wind and the momentum flux is conserved, a mass of $4 M_{\odot}$ can be dispersed by an early-type star in $1 \mathrm{Myr}$, whereas the same amount will be dispersed by a late-type star in $10 \mathrm{Myr}$. These rates are consistent with our data in early- and late-type stars for ages between $0.1 \mathrm{Myr}$ and $1 \mathrm{Myr}$. The change of $\alpha$ to positive values during this period shows that the feeding of the circumstellar disk by the cloud material has stopped or, at least, the accretion rate has decreased below the mass dispersal rate.

Photodissociation becomes very efficient in early-type stars (Hollenbach et al. 2000). If photodissociation is the mechanism responsible for the disruption of the parent core, the correlation between cloud morphology and spectral type (or luminosity) of Type III stars can be easily explained. In fact, Díaz-Miller et al. (1998) show that only stars with spectral type earlier than B5 can create prominent photodissociation regions (PDRs) (for example, $R_{\mathrm{PDR}}=0.01 \mathrm{pc}$ for an initial density $\left.n=10^{5} \mathrm{~cm}^{-3}\right)$. This cutoff roughly coincides with our division of earlyand late-type stars, and could explain the different dispersal efficiency between early- and late-type stars in the later stages of the PMS evolution. This mechanism can explain the larger mass dispersal rate after $\sim 1 \mathrm{Myr}$ in early-type stars. In fact, it is in 1 Myr that a B5 star $\left(\sim 6 M_{\odot}\right)$ is expected to meet the ZAMS. At that point, the star is hot enough to start creating the PDR immediately. The extent and time-scale for the formation of the PDR is strongly dependent on the density. If the dense gas has been dispersed in an earlier evolutionary stage (bipolar outflows, accretion, stellar winds), the PDR expands

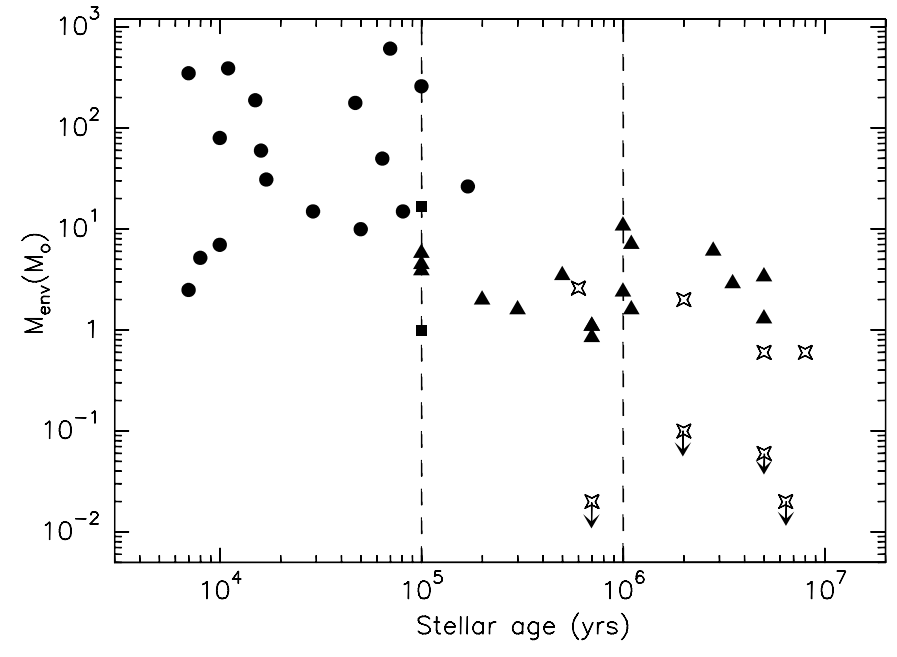

Fig. 10. Circumstellar mass vs. stellar ages for protostars (filled circles), Type I (filled squares), Type II (filled triangles) and Type III (open stars) HAEBE stars.

rapidly in a low density medium $\left(\$ 10^{3} \mathrm{~cm}^{-3}\right)$, creating a large cavity in a timescale of $\sim 1 \mathrm{Myr}$.

\section{Summary: the history of the circumstellar material around intermediate mass objects}

In Fig. 10 we have plotted the circumstellar mass as a function of the stellar age for the sample of HAEBE stars and protostars listed in Tables 1 and 2. The number of stars plotted in this Figure is lower than the number of stars listed in Table 1 because we do not have a reliable age estimate for all the sample. In the case of protostars, the adopted age is the kinematical age of the outflow. Protostars are drawn as filled circles, Type I stars as filled squares, Type II stars as filled triangles and Type III stars as empty stars. Both, early-type and late-type objects have been represented. Three different regions which are marked with dashed lines can be distinguished in this plot. For ages $<0.1$ Myr there does not exist any correlation of the circumstellar mass with the stellar age. A dispersion of almost 3 orders of magnitude is found in the circumstellar masses. All the objects in this region are protostars. The circumstellar mass in this case is determined by the luminosity of the source rather than by the stellar age. From 0.1 to $\gtrsim 1 \mathrm{Myr}$, the circumstellar mass remains constant with values ranging between 1-10 $M_{\odot}$ for all the stars. For ages $>1$ Myr, we find again a large dispersion (at least two orders of magnitude) in the circumstellar masses. Some stars are still Type II stars while others are Type III stars and have completely dispersed the circumstellar material.

This behavior can be easily explained in terms of the different timescales for the evolution of late-type and early-type stars, and the different mass dispersal mechanisms at work at the different evolutionary stages. Assuming a typical accretion rate of $10^{-5} M_{\odot} \mathrm{yr}^{-1}$ for protostars, a late-type star $\left(2-4 M_{\odot}\right)$ will be formed in 
a few 0.1 Myr. By this time, about $90 \%$ of the circumstellar dense core has been dispersed by bipolar outflows. From $\sim$ a few 0.1 to $\geq 1 \mathrm{Myr}$, the star undergoes the PMS contraction. Small material is removed during this phase because the outflow fades and the star is still too cold to generate UV photons. The stellar wind is the only dispersal mechanism at work during this phase. From $\geq 1$ to 10 Myr the star meets the ZAMS and start excavating the cloud by UV radiation but at a very low rate in the case of late-type stars. Because of their low effective temperature, late-type stars are unable to excavate large cavities.

Assuming the same accretion rate for early-type and late-type stars, in a typical time $\leq 1$ Myr a star of $6-8 M_{\odot}$ has been formed and almost the entire dense core has been dispersed by bipolar outflows. In $1 \mathrm{Myr}$, the early-type star meets the ZAMS and starts creating a cavity in the molecular cloud. Because of the large flux of photodissociating photons and the strong ionized winds, these stars can create large cavities in a timescale of $\sim 1$ Myr. This can explain the large number of Type III early-type stars found in our sample (compared with Type III late-type stars) as well as the different morphology of the material surrounding early-type and late-type Type III stars.

Acknowledgements. This work has been partially supported by the Spanish DGES under grant AYA2000-927 and Spanish CICYT-PNIE under grant 1FD1997-1442.

\section{References}

Bachiller, R., Fuente, A., \& Kumar, M. S. N. 2002, A\&A, 381, 168

Bechis, K. P., Harvey, P. M., Campbell, M. F., \& Hoffmann, W. F. 1978, ApJ, 226, 439

Berrilli, F., Corciulo, G., Ingrosso, G., et al. 1992, ApJ, 398, 254

Bevington, P. R. 1969, Data Reduction and Error Analysis for the Physical Sciences (ed. Mc-Graw-Hill, Inc.), 180

Brand, J., Cesaroni, R., Palla, F., \& Molinari, S. 2001, A\&A, 370,230

Cabrit, S., \& Andre, P. 1991, ApJ, 379, L25

Cabrit, S., \& Bertout, C. 1992, A\&A, 261, 274

Cesaroni, R., Felli, M., Jenness, T., et al. 1999, A\&A, 345, 949

Codella, C., \& Bachiller, R. 1999, A\&A, 350, 659

Chandler, C. J., \& Richer, J. S. 2000, ApJ, 530, 851

Davidson, J. A. 1987, ApJ, 315, 602

Díaz-Miller, R. I., Franco, J., \& Shore, S. N. 1998, ApJ, 501, 192

Dickman, R. L. 1978, ApJS, 37, 407

Eiroa, C., Palacios, J., \& Casali, M. M. 1998, A\&A, 335, 243

Font, A. S., Mitchell, G. F., \& Sandell, G. 2001, ApJ, 555, 950
Fuente, A., Martín-Pintado, J., Bachiller, R., Neri, R., \& Palla, F. 1998, A\&A, 334, 253 (Paper I)

Fuente, A., Neri, R., Martín-Pintado, J., et al. 2001, ApJ, 366, 873

Hasegawa, T. I., \& Mitchell, G. F. 1995, ApJ, 451, 225

Herbig, G. H., \& Bell, K. R. 1988, Lick Obs. Bull., No. 1111

Hillenbrand, L. A., Strom, S. E., Vrba, F. J., \& Keene, J. 1992, ApJ, 397, 613

Hillenbrand, L. A. 1995, Ph.D. Thesis, University of Massachusetts Amherst

Hog, E., Kuzmin, A., Bastian, U., et al. 1998, A\&A, 335, L65

Hollenbach, D. J., Yorke, H. W., \& Johnstone, D. 2000, in Protostars and Planets IV, ed. V. Mannings, A. Boss, \& S.S. Russell (Tucson: University of Arizona Press), 401

Ladd, E. F., Fuller, G. A., \& Deane, J. R. 1998, ApJ, 497, 871

Launhardt, R., Ward-Thompson, D., \& Henning, Th. 1997, MNRAS, 288, L45

Margulis, M., \& Lada, C. J. 1985, ApJ, 299, 925

Molinari, S., Testi, L., Brand, J., Cesaroni, R., \& Palla, F. 1998, ApJ, 505, L39

Motte, F., Andre, P. \& Neri, R. 1998, A\&A, 336, 150

Mundy, L. G., Wilking, B. A., \& Myers, S. T. 1986, ApJ, 311, L75

Myers, P. C., Heyer, M., Snell, R. L., \& Goldsmith, P. F. 1988, ApJ, 324, 907

Palla, F., \& Stahler, S. W. 1993, ApJ, 418, 414

Perryman, M. A. C., Lindegren, L., Kovalevsky, J., et al. 1997, A\&A, 323, L49

Sandell, G., Aspin, C., Duncan, W. D., Robson, E. I., \& Dent, W. R. F. 1990, A\&A, 232, 347

Sandell, G., \& Weintraub, D. A. 1994, A\&A, 292, L1

Shepherd, D. S., Watson, A. M., Sargent, A. I., \& Churchwell, E. 1998, ApJ, 507, 861

Shepherd, D. S., Yu, K. C., Bally, J., \& Testi, L. 2000, ApJ, 535,833

Skinner, S. L., Brown, A., \& Stewart, R. T. 1993, ApJS, 87, 217

Skinner, S. L. 1994, in The nature and evolution of Herbig Ae/Be stars, ed. P. S. The, M. R. Perez, \& E. P. J. Van den Heuvel (San Francisco: ASP), 143

Snell, R. L., Scoville, N. Z., Sanders, B., \& Erickson, N. R. 1984, ApJ, 284, 176

Tafalla, M, Myers, P. C., Caselli, P., Walmsley, C. M., \& Comito, C. 2002, ApJ, in press

Testi, L., Palla, F., \& Natta, A. 1998, A\&AS, 133, 81

Testi, L., Sargent, A. I., Olmi, L., \& Onello, J. S. 2000, ApJ, 540, L53

Thé, P. S., de Winter, D., \& Pérez, M. R. 1994, A\&AS, 104, 315

van den Ancker, M. E., de Winter, D., \& Tjin A Djie, H. R. E. 1998, A\&A, 330, 145

Velusamy, T., \& Langer, W. D. 1998, Nature, 392, 685

Yamashita, T., Suzuki, H., Kaifu, N., et al. 1989, ApJ, 347, 894 